\title{
SRMS History, Evolution and Lessons Learned
}

\author{
Glenn Jorgensen ${ }^{1}$ and Elizabeth Bains. ${ }^{2}$ \\ National Aeronautics and Space Administration
}

\begin{abstract}
Early in the development of the Space Shuttle, it became clear that NASA needed a method of deploying and retrieving payloads from the payload bay. The Shuttle Remote Manipulator System (SRMS) was developed to fill this need. The $\mathbf{5 0}$ foot long robotic arm is an anthropomorphic design consisting of three electromechanical joints, six degrees of freedom, and two boom segments. Its composite boom construction provided a light weight solution needed for space operations. Additionally, a method of capturing payloads with the arm was required and a unique End Effector was developed using an electromechanical snare mechanism. The SRMS is operated using a Displays and Controls Panel and hand controllers located within the aft crew compartment of the shuttle. Although the SRMS was originally conceived to deploy and retrieve payloads, its generic capabilities allowed it to perform many other functions not originally conceived of. Over the years it has been used for deploying and retrieving constrained and free flying payloads, maneuvering and supporting EVA astronauts, satellite repair, International Space Station construction, and as a viewing aid for on-orbit International Space Station operations. After the Columbia accident, a robotically compatible Orbiter Boom Sensor System (OBSS) was developed and used in conjunction with the SRMS to scan the Thermal Protection System (TPS) of the shuttle. These scans ensure there is not a breach of the TPS prior to shuttle re-entry. Ground operations and pre mission simulation, analysis and planning played a major role in the success of the SRMS program. A Systems Engineering Simulator (SES) was developed to provide a utility complimentary to open loop engineering simulations. This system provided a closed-loop real-time pilot-driven simulation giving visual feedback, display and control panel interaction, and integration with other vehicle systems, such as GN\&C. It has been useful for many more applications than traditional training. Evolution of the simulations, guided by the Math Model Working Group, showed the utility of input from multiple modeling groups with a structured forum for discussion.There were many unique development challenges in the areas of hardware, software, certification, modeling and simulation. Over the years, upgrades and enhancements were implemented to increase the capability, performance and safety of the SRMS. The history and evolution of the SRMS program provided many lessons learned that can be used for future space robotic systems.
\end{abstract}

\footnotetext{
${ }^{1}$ Shuttle Division Chief Engineer for the, Automation, Robotics and Simulation Divison, NASA-JSC, 2101 NASA Parkway, Houston,77058.

${ }^{2}$ Integrated Analysis Manager, Automation, Robotics and Simulation Divison, NASA-JSC, 2101 NASA Parkway, Houston,77058 and AIAA Associate Fellow.
} 


\section{System Description and Evolution of the Shuttle Remote Manipulator System}

$\mathrm{T}$

HE fundamental requirement of the Shuttle Remote Manipulator System (SRMS) was to deploy and retrieve payloads to and from space. To accomplish this mission, the system that was developed consisted of an anthropomorphic manipulator arm located in the shuttle cargo bay (shown in Figure 1), cabin equipment to provide an interface to the main shuttle computer, and a human interface to allow an astronaut to control arm operations remotely.

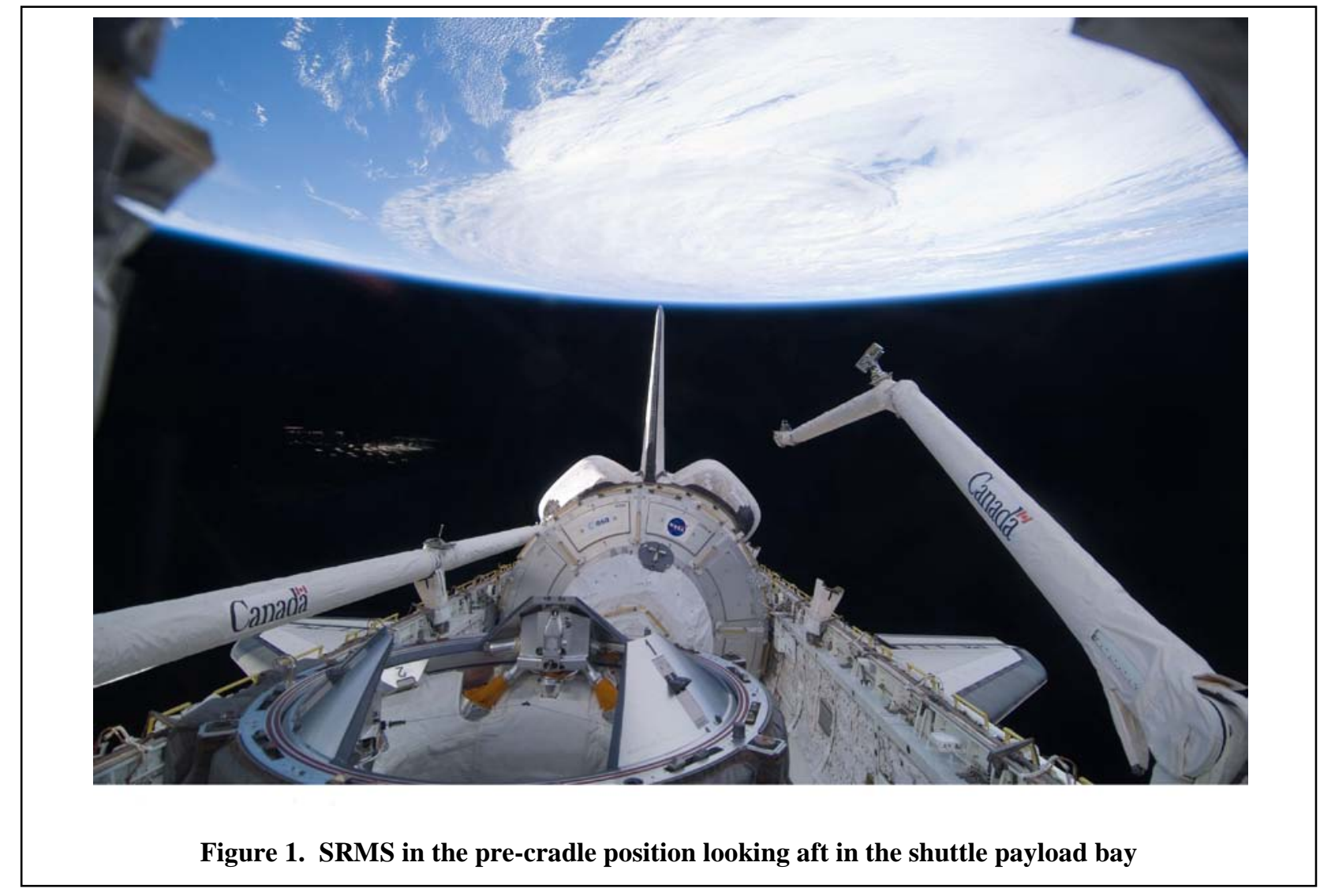

\section{A. Hardware Overview}

The manipulator arm consists of three joints, two arm booms, an end effector, a Thermal Protection System, and a closed-circuit television system. Figure 2 breaks down the primary SRMS components. The arm joints included a shoulder joint with two degrees of freedom (yaw and pitch), an elbow joint with one degree of freedom (pitch), and a wrist joint with three degrees of freedom (pitch, yaw, and roll). Each joint degree of freedom consists of a motor module driving a gear box to effect joint movement and appropriate local processing to interpret drive commands originating from the cabin electronics.

The cabin electronics includes a displays and controls subsystem that provides the human-machine interface to allow a crew member to command the arm and view displays of appropriate information, including arm position and velocity, end effector status, temperature, and caution and warning information. Additionally, in the displays and controls subsystem, two hand controllers allow man-in the-loop control of the end point of the arm. The main arm processor is the manipulator controller interface unit (MCIU) that is also part of the cabin electronics. It handles all data transfer between the arm, displays and controls panel, and the main shuttle computer. The main shuttle computer processes commands from the operator via the displays and controls panel; receives arm data to determine real-time position, orientation, and velocity; and then generates rate and current limit commands that are sent to the arm-based electronics. The arm is thermally protected with specially designed blankets to reduce the susceptibility 
of the hardware to the thermal extremes experienced during spaceflight and has an active thermostatically controlled and redundant heater system.

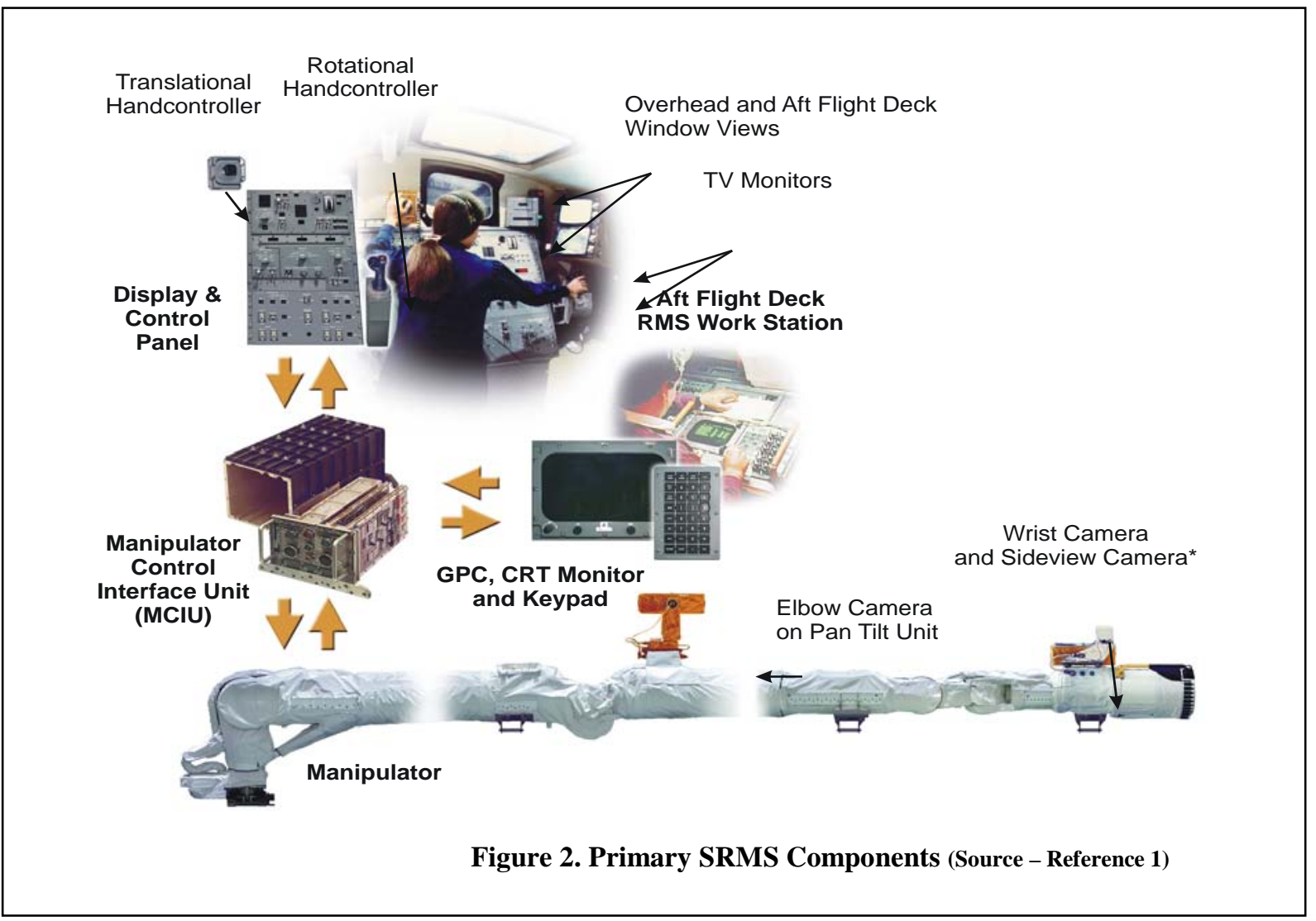

The closed-circuit television system consists of three cameras. A color camera on a pan/tilt unit near the elbow joint was used as a situational awareness camera for the SRMS operator. A second camera in a fixed location on the wrist joint was primarily used to view a grapple fixture target when the arm was capturing a payload. A third camera called the SRMS Side-view Camera was added after the Columbia accident. This was fixed on the inboard side of the wrist joint and was used as a situational awareness camera when the SRMS was performing Shuttle Thermal Protection System inspections with the Orbiter Boom Sensor System (discussed later).

Self-checks exist throughout all the Shuttle Robotic Arm electronics to assess arm performance and apply appropriate commands to stop the arm, should a failure occur. Caution and warning displays provide the operator with insight into the cause of the failure and remaining capability to facilitate the development of a workaround plan.

\section{B. SRMS Operating Modes}

The operating modes of the SRMS included Automatic modes, Manual Augmented modes, Single drive, Direct drive and a Back-up drive. Auto, Manual Augmented, and Single drive mode were supported with the main Shuttle computer and included servo joint rate control. Maximum joint rates were determined pre-flight and varied based on the payloads being handled. Direct and Back-up drive modes were hardwired, reduced capability modes that applied a fixed voltage to the joint drive amplifier.

Automatic modes included pre-programmed and operator commanded auto sequences. The pre-programmed sequences were developed prior to flight and loaded into the main shuttle computer. The operator commanded auto sequence mode required the operator to input a final SRMS position and orientation before arm motion was initiated. Auto sequences were used primarily for shuttle and payload surveys and lengthy repositioning maneuvers. 
Manual Augmented modes allowed the SRMS operator to manipulate the SRMS position and orientation real time using the Translational and Rotational hand controllers. The Point of Resolution and co-ordinate axes could be varied depending on the operation being performed. This mode was used for grappling, berthing and un-berthing payloads, track and capture of free flying payloads, and coarse positioning of the SRMS.

Single joint drive allowed the operator to control each joint individually. This mode was used primarily to cradle and un-cradle the arm, and for fine positioning of the SRMS.

Direct and Back-up drive modes were used for contingency arm motion should computer supported modes be lost. They allowed only single joint control.

\section{The SRMS End Effector}

The interfacing end of the Shuttle Robotic Arm is equipped with an electromechanical End Effector. Figure 3 shows a picture of the End Effector on the end of the SRMS wrist joint. The wrist camera/light assembly is also shown. The End Effector is the equivalent to a human hand and is used to grapple a payload fitted with a specially designed interface known as a grapple fixture. A picture of a grapple fixture is shown in Figure 4.

The grapple fixture includes a grapple pin, a target, and three alignment cams that mate to cam lobes on the End Effector. The End Effector uses a three cable snare mechanism that closes around the grapple pin (see Figure 5). Once grappled to the pin, the snare mechanism is drawn inward into the main body of the End Effector to "rigidize" the payload to the SRMS. The cams ensure there is no End Effector/Grapple fixture motion once the payload is captured.

The standard grapple fixture is called a Flight Releasable Grapple Fixture, since it is equipped with a mechanism that can be activated by an EVA crewman to release the grapple pin should the End Effector fail while grappled to a payload.

The End Effector is also equipped with an electrical connector called a Special Purpose End Effector (SPEE) connector. This connector is fed with sixteen power and data lines from a cable harness routed along the length of the SRMS. This connector can mate to a specially designed grapple fixture called an Electrical Flight Grapple Fixture (EFGF). When captured, a payload equipped with an EFGF can be sent power and data signals through the SRMS cable harness. A switch panel within the Shuttle was configured to provide these signals.

To track and capture a payload required the use of the grapple fixture target in conjunction with SRMS wrist camera. The camera is oriented to allow a straight on view of the target. The camera video was displayed to the crew in the aft 
flight deck, and helped the crew properly position the end effector relative to the grapple fixture prior to capturing a payload. An overlay on the CRT display was used to aid the crewman in determining when the End Effector was over the grapple pin and ready for capture. The SRMS was maneuvered to position the camera view of the grapple fixture target within the boundaries of the overlay. When satisfied with the relative position of the End Effector to the grapple fixture target, the crew used a switch on the Rotational Hand Controller to capture and secure the payload. The End Effector could be operated in either automatic or manual modes. In automatic mode the rigidize command would be initiated automatically after the snares were closed around the grapple pin. This was the standard mode of operation. Manual mode required the crewman to input the snare close and rigidize commands separately.

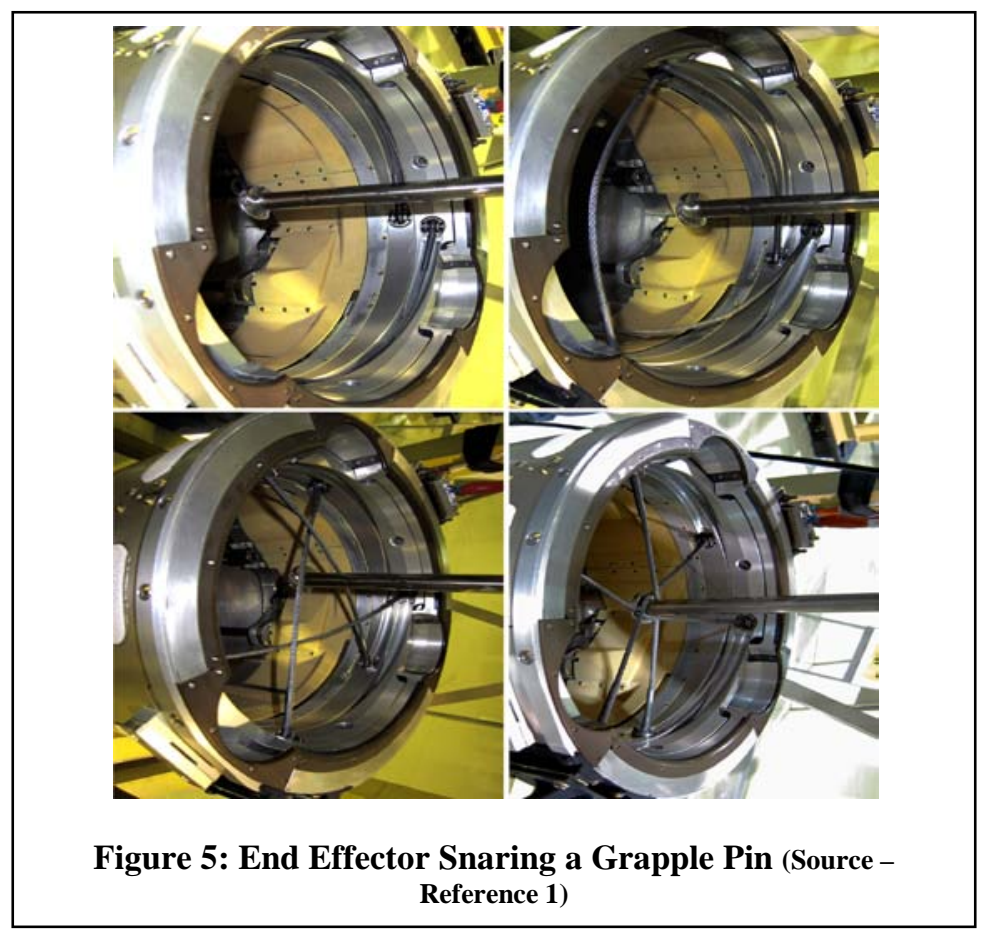

\section{SRMS History of Operations}

The SRMS first flew on STS-2, and by the end of the Shuttle program it had flown a total of ninety-one missions. While originally envisioned only for satellite deployment and retrieval, the utility of a robotic arm for other tasks quickly became clear. Over the life of the program the SRMS evolved into an indispensible tool not only for satellite deployment and retrieval, but was used extensively for satellite rescue and repair, as an EVA platform, International Space Station construction, shuttle and payload bay surveys, as well as a host of contingency operations.

There were only three on orbit failures in the history of the SRMS program and only one of these failures prevented completion of the mission. This was STS-41B when the wrist yaw motor commutator failed causing loss of all primary modes of operation. The payload scheduled to be deployed (the SPAS satellite) had to remain in the payload bay. The second failure was on STS-51I when the power to the elbow joint electronics was shorted to ground. The crew managed to complete payload operations using back-up drive for the elbow joint and single joint drive for the other joints. The third failure was due to an incorrectly installed SPEE connector that prevented power and data transfer to the payload (EURECA satellite). The satellite was berthed and the data transfer took place via a payload bay connector.

Other less significant failures were experienced, and these were all worked by the ground team and the crew to allow completion of the mission with no loss of functionality in the SRMS. The reliability of the SRMS in its thirty year history is testament to the excellent design, manufacturing and testing teams, and to the ground support personnel working with the rest of the shuttle team. The SRMS has proven its worth and has provided a blue print for future robotic arms in space.

\section{A. Satellite Deployment, Retrieval, and Servicing}

The list of satellites that were deployed and retrieved by the SRMS is long and impressive. The heavy lift capability of the Shuttle combined with the capabilty of the SRMS allowed unique satellite missions to be designed that were not previously possible. The retrieving capability of the SRMS made on-orbit repair and maintenance of satellites a reality for the first time.

Figure 6 is a picture of the SRMS deploying the Gamma Ray Observatory (GRO) on STS-37. Other payloads of note deployed by the SRMS include the Earth Radiation Budget Satellite (ERBS) on STS-41G, and the Upper Atmosphere Research Satellite (UARS) on STS-48. 
The majority of payloads deployed were also retrieved by the SRMS. The first successful capture of a free flying payload was on STS-7 when the SPAS-1 satellite, originally deployed earlier in the mission, was captured and berthed in the payload bay by the SRMS. Other deployments and captures include SPARTAN on STS-51G, the Plasma Diagnostics Package (PDP) on STS-51F, and the SPAS-2 payload on STS-39. The Long Duration Exposure Facility (LDEF) was deployed on STS-41C and retrieved on STS32 (see Figure 7). The European Retrievable Carrier satellite (EURECA) was deployed on STS-46 and retrieved on STS-57.

The SRMS also played the primary role in rescuing malfunctioning satellites. For example, during STS-41C, it was used to grapple and berth the spinning Solar Maximum Mission satellite (Solar Max). It had been deployed in 1980 and had been fitted with a grapple fixture to allow capture with the SRMS. The track and capture capability of the SRMS was put to the test due to the high spin rate of Solar Max. The SRMS was used on STS-51A during the retrieval of two malfunctioning comsats, Palapa B2 and Westar VI, which were subsequently refurbished on Earth and reflown. One of the more notable SRMS accomplishments was the deployment of the Hubble Space Telescope (HST) on STS-31 in April, 1990. This was followed by four successful HST servicing missions (STS-61 in 1993, STS-103 in 1999, STS-109 in 2002, and STS-125 in 2009) during which the SRMS was used to retrieve, repair and redeploy the space telescope. The final servicing mission, STS-125, is expected to extend the life of the HST to 2014.

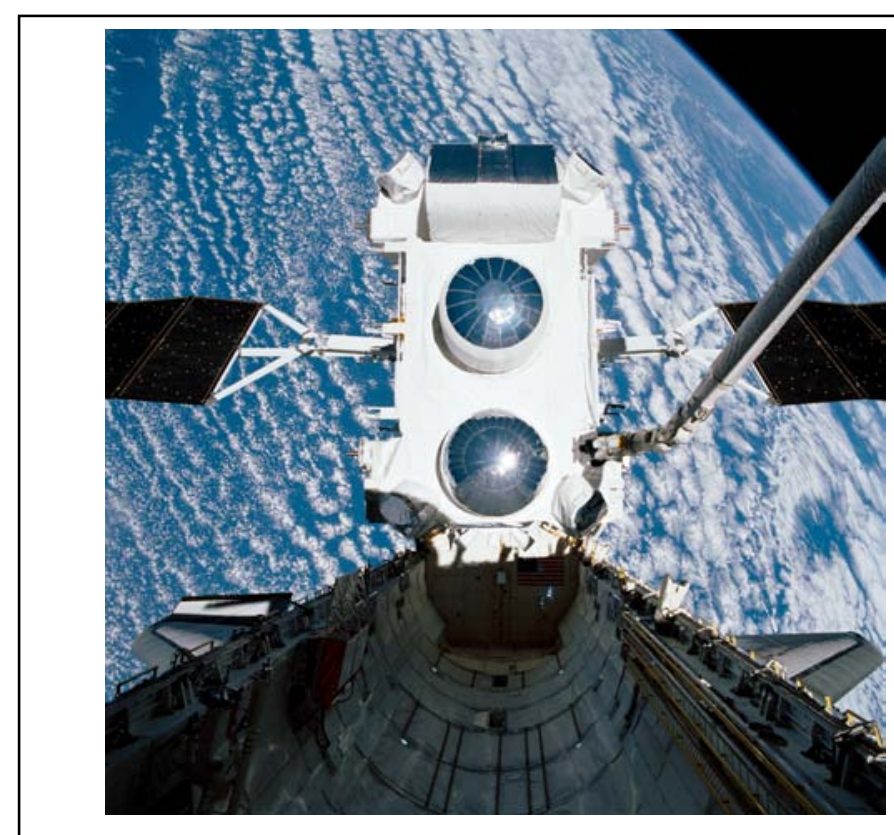

Figure 6. STS-37 - Deploying the Gamma Ray Observatory

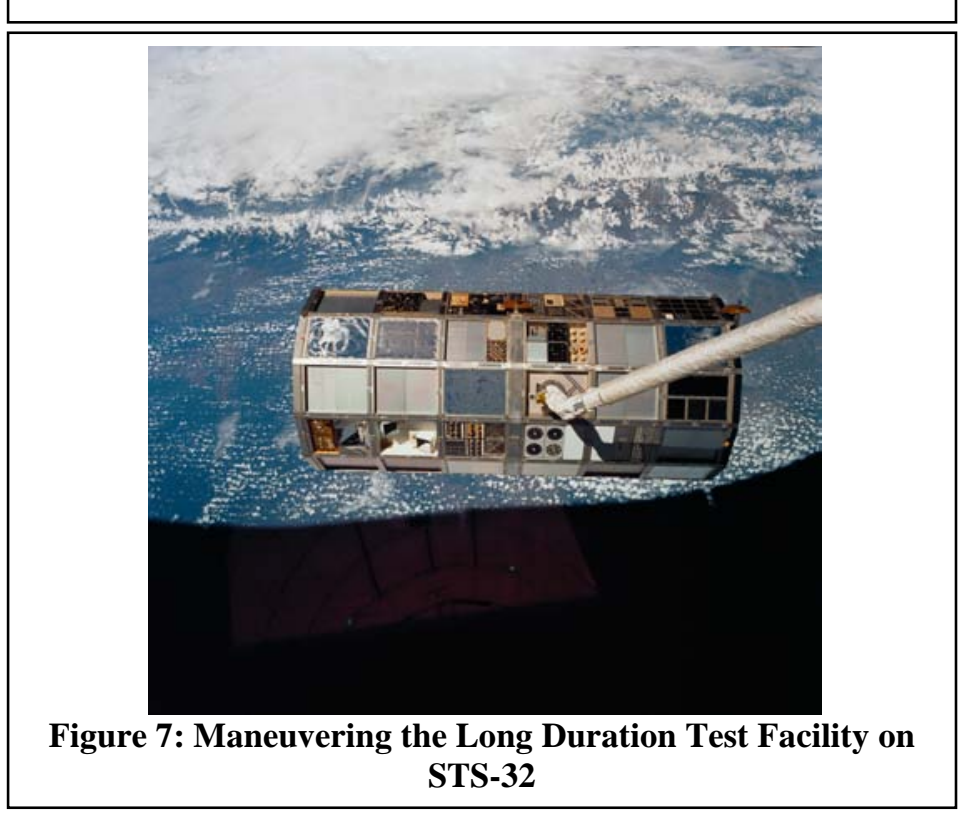

\section{B. Extra Vehicular Activity (EVA) Platform}

A mobile work platform for EVA astronauts became one of the most important uses for the SRMS. The arm was used extensively in this capacity, both for pre-planned operations and for contingency EVA's planned real-time that were needed for inspection or repair. The EVA crewmember could be mounted on a portable foot restraint attached directly to the SRMS, or he could be mounted to the Manipulator Foot Restraint (MFR) that was grappled by the End Effector, or the crewman could simply grab the EVA handhold attached to the End Effector. The arm operator inside the shuttle could easily position the EVA crewmember anywhere within the reach capability of the arm. The stable platform provided by the SRMS allowed the crewman to perform tasks much easier than if he were free floating. Radio communication between the EVA crewman and the arm operator enabled quick repositioning as 
required. Tool stanchions and holders were developed to aid in the repair and maintenance activites. There are many examples during the life of the Shuttle program where the SRMS was used to support EVA, but none provide more concrete evidence of the value of a robotic arm as an EVA platform than the HST repair missions.

The Hubble Space Telescope, deployed on STS-31 (1990), gave the world a perspective of outer space never before seen. The first servicing mission repaired a problem with the telescope's main lens assembly. The subsequent missions replaced and enhanced onboard instrumentation. This improved the accuracy and extended the life of HST. Figure 8 shows an EVA crewman on the end of the SRMS preparing for a Hubble repair task. More than any other payload, the Hubble servicing missions displayed to the world the unique capabilities of the SRMS.

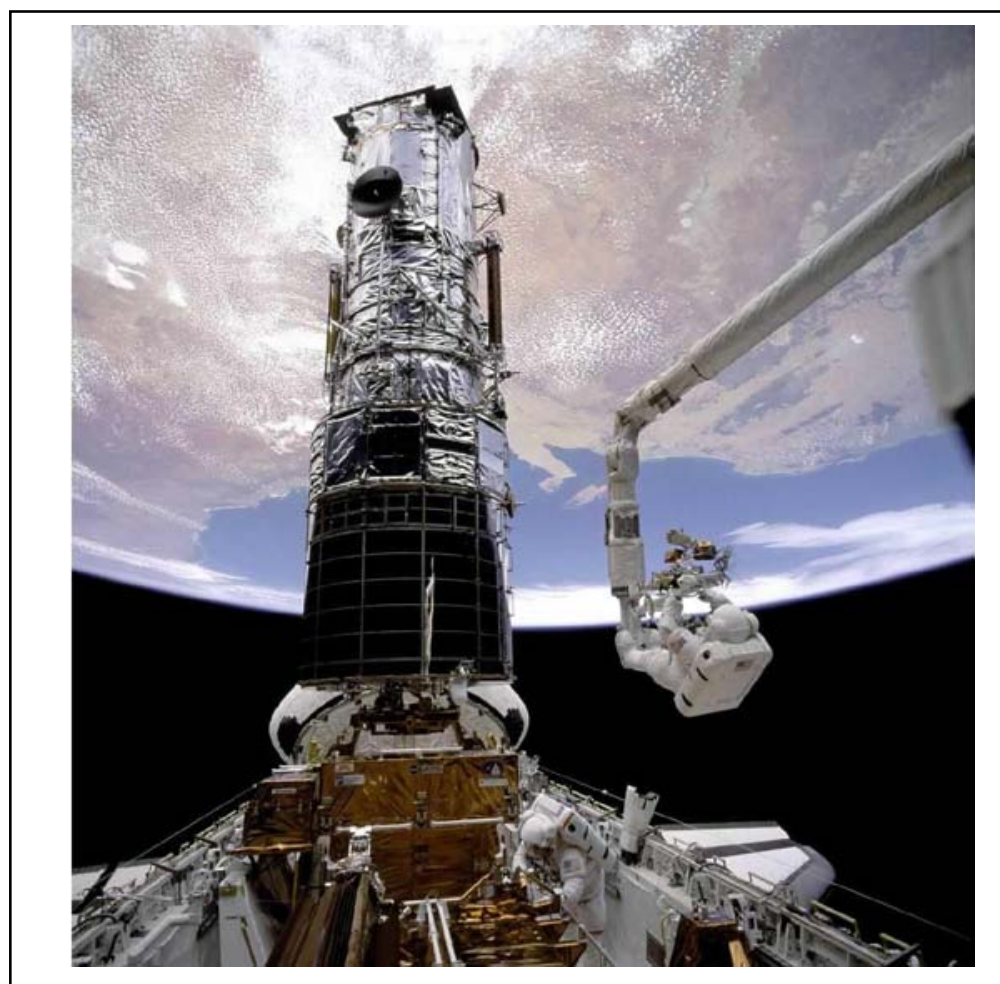

Figure 8: Hubble Space Telescope EVA Repair Work on STS-61

\section{The Era of Space Station Construction}

The SRMS was the primary mechanism used for early International Space Station (ISS) construction. During the later stages when the ISS was so large that the SRMS could not reach many berthing locations, the SRMS was used in conjunction with the Space Station Remote Manipulator System (SSRMS) to complete the construction. In fact, the SRMS served as the proof of concept for the SSRMS, and many of the design features, capabilities and operations of the SRMS were used in the design and development of the SSRMS. Figure 9 shows the ISS P5 Truss segment being handed off on STS105. ISS elements that were handled by the SRMS included Nodes 1,2 and 3, the US Laboratory Module the Space Station Airlock, nine truss segments, many of which contained solar

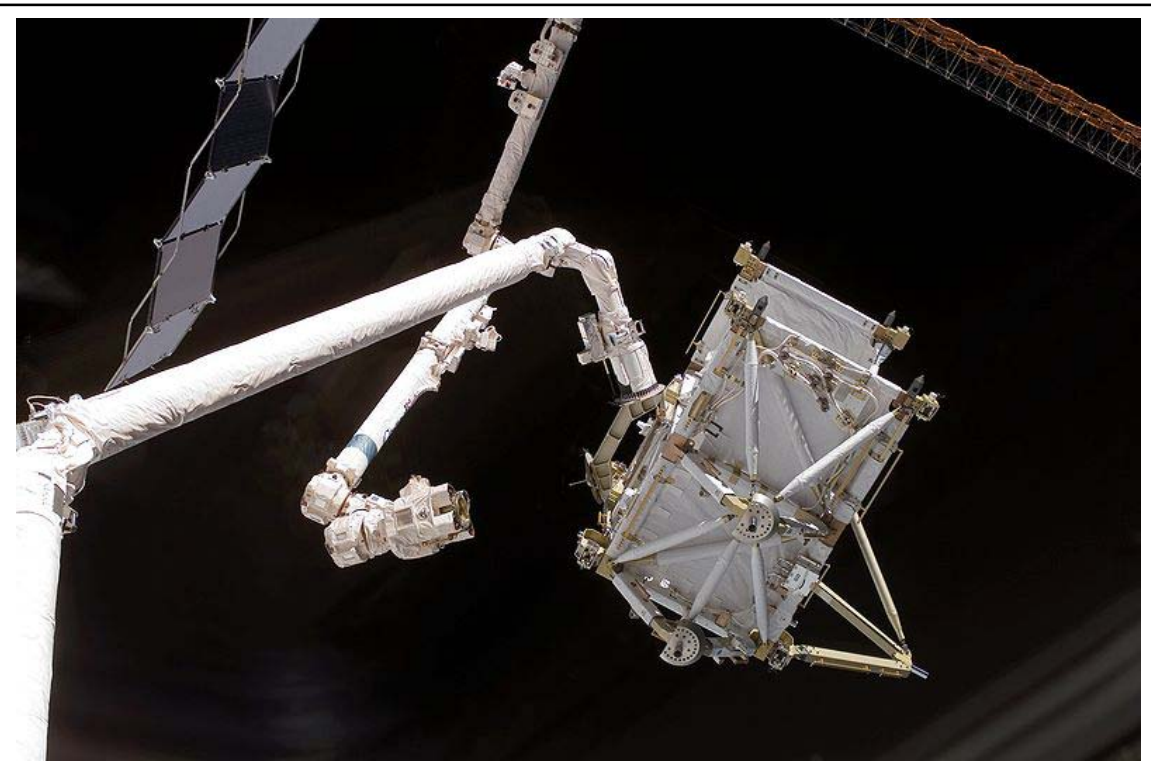

Figure 9: Handing off the ISS P5 Truss Segment from SRMS to SSRMS

panels for power to the station; and the Space Station Remote Manipulator System (SSRMS). These activities required some modifications to the SRMS itself, as well as the addition of systems to enhance alignment and 
berthing operations. During the preliminary planning, studies evaluated the adequacy of the SRMS to handle the anticipated payload operations envisioned for the space station construction. These studies determined that arm controllability would not be satisfactory for the massive payloads the arm would need to manipulate. Redesigning the Servo Power Amplifiers (part of the Arm Based Electronics) in each joint provided the necessary controllability. Also, the addition of increased self-checks assured better control of hardware failures that could cause hazardous onorbit conditions. During the process of assembling the space station, enhanced berthing cue systems were necessary to mate complicated interfaces that would need to transmit loads and maintain a pressurized interior. The complexity and close tolerance of mating parts led to the development of several berthing cue systems, such as the space vision system and the centerline berthing cue system, to enhance the crew's ability to determine relative position between mating modules.

\section{Return to Flight After the Columbia Accident - The Orbiter Boom Sensor System}

During the launch of the Space Shuttle Columbia on STS-107 (2003), a piece of debris hit the shuttle, causing a rupture in the Thermal Protection System that is necessary for re-entry into Earth's atmosphere, thereby leading to the Columbia accident. The ramifications of this breach in the shuttle's Thermal Protection System changed the role of the SRMS substantially for all post-Columbia accident missions. Development of the robotically compatible 50-ft Orbiter Boom Sensor System (OBSS), carried on the starboard mai=nipulator positioning mechanisms, provided a shuttle inspection and repair capability that addressed the Thermal Protection System inspection requirement for post-Columbia Return to Flight missions. Figure 10 shows the OBSS grappled by the SRMS.

Modification of the robotic arm wiring provided power and data capabilities to support the inspection cameras and lasers at the tip of the inspection boom. Two shuttle repair capabilities were provided in support of the Return to Flight effort. The first scenario required the SRMS, grappled to the space station, to position the shuttle and the space station in a configuration that would enable a crew member on the Space

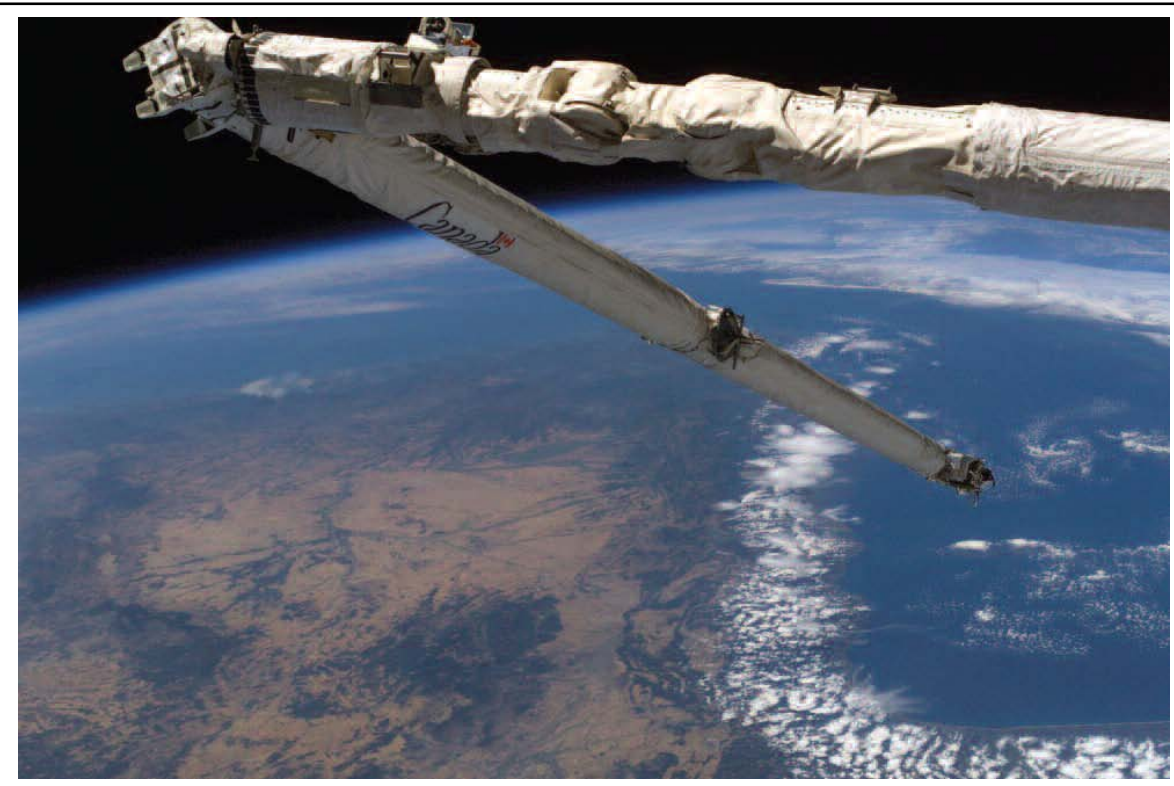

Figure 10: The SRMS Grappled to the Orbiter Boom Sensor System

Station Robotic Arm to perform a repair. This was referred to as the Orbiter Repair Maneuver (ORM). The second repair scenario involved the Shuttle Robotic Arm holding the boom with the astronaut at the tip.

All of the post-Columbia-accident missions employed the SRMS and Orbiter Boom Sensor System combination to survey the shuttle for damage. The SRMS and OBSS were used to inspect all critical Thermal Protection System surfaces. After the imagery data were processed, focused inspections occasionally followed to obtain additional images of areas deemed questionable from the inspection. A detailed test objective on STS-121 (2006) demonstrated the feasibility of having a crew member on the end of the OBSS grappled by the SRMS and performing actions similar to those necessary for Thermal Protection System repair. Test results showed that the integrated system could be used as a repair platform and that the system was controllable with the correct control parameters, good crew training, and proper extravehicular activity procedures development.

In support of shuttle repair capability using the Orbiter Repair Maneuver described earlier, the simulation model of the SRMS was updated to evaluate the handling of the space station as a "payload". This heavy payload model update proved useful when it was decided to repair the Hubble Space Telescope on STS-125. This is because after 
the Columbia accident, a method of crew rescue was mandatory for all shuttle flights. A "Launch on Need" shuttle was manifested for crew rescue should the shuttle on orbit sustain damage that would prevent re-entry. Given that the space station would not be available for crew rescue for the final Hubble servicing mission, the SRMS would be used to grapple the failed Shuttle. For the crew from the disabled shuttle to get to the rescue shuttle, the Shuttle Robotic Arm would act as a path for crewmen to transfer between the two vehicles. The updated SRMS simulation for heavy payloads proved its worth when developing and validating this rescue scenario. Fortunately, neither of these repair/rescue capabilities_-Orbiter repair maneuver or Hubble rescue — ever had to be used.

\section{Unique Challenges in the Development of the SRMS}

\section{A. Mechanical Design}

As with all space systems, a tradeoff between mass and strength/stiffness had to be considered in the mechanical design of the SRMS. With stiffness a primary consideration along with low mass, the material chosen for the booms of the SRMS was a carbon composite material. In addition to stiffness, the high strength to weight ratio of carbon composite makes it ideal for space applications.

Additionally stiffness was the driver in the design of the joints. The elbow joint, having only one degree of freedom, could not relieve forces and moments acting in off-axis directions, so strength was also a primary driver in its design. The joint construction was primarily fabricated aluminum housings, with some steel and titanium for higher load bearing components. The gears were made from Carpenter Custom 455 steel, an exceptionally high strength material (reference 1).

The joint actuation design was electro-mechanical using brushless DC motors and high ratio gear boxes. Identical motors were used in every joint to minimize qualification requirements. Hydraulic joints were considered however the potential for contamination from hydraulic fluid leakage eliminated this option. The choice to use relatively small motors driving the joint through a gear box, instead of larger motors in a direct drive configuration, was driven by the need to reduce weight. The gear ratios were adjusted based on the motor torque characteristics, and the requirement for high forward and backdrive efficiency, low backlash, and high drive train stiffness (reference 1).

Each gearbox was designed with two stages. The first stage was a high speed design and the gear reduction ratio varied from joint to joint to meet the specific joint torque requirement. The second stage was identical in all joints and was a planetary design that maximized the number of load paths and provided high stiffness and low backlash.

Backdrivability was a key driver in the design of the joints. High external loads can result in forces and moments applied to the arm that exceed its structural capability. Joints that can be backdriven will reduce the potential for this excessive loading. The back drivability requirement drove the need for high gearbox efficiencies.

The efficiency and back drivability requirements led to problems in the manufacture of the gearboxes. Tight tolerances in manufacture are necessary to achieve the degree of backlash and friction required to meet the control system requirements. Gears had to be chosen based on a "select-on-manufacture" program resulting in a low yield rate and higher costs.

To ensure the joints did not apply excessive loads when the joints were in forward drive mode, motor current limiting was implemented in the servo power amplifiers that provided the electrical power to the motors. The forward and backdrive current limits were matched to the gearbox effieciencies. Thus dynamic conditions were similar in all four quadrants of the drive system.

The joint motor was chosen to minimize weight and eliminate maintenance. The brushless design avoided the wear and tear of commutator brushes. The motor chosen was a frameless design, so it was assembled in a housing that included the motor and commutator, plus a brake and a tachometer. The entire unit was called the motor module and was common to all six joints. To ensure servo stability when the motor was driving in a no load condition, a flywheel was added.

Dynamic braking using the motor to decelerate the joint was also incorporated into the joint design. This eliminated the requirement to apply mechanical brakes each time a drive command was removed and prevented excessive wear on the brakes. This was required to meet the life requirements (originally 10 years but extended as the program evolved).

The SRMS electronics had extensive Built-In Test Equipment (BITE) that detected potentially catastrophic failures and automatically applied brakes to prevent joint runaway and inadvertent contact between the arm and external shuttle/payload structure. To reduce the potential to exceed the structural load limits of the arm under these malfunction conditions, the joints had to be backdrivable with the brakes applied. Thus the brakes had to be 
designed not only to provide a minimum holding torque, but also a maximum torque. It was found that the holding torque of the original brake material (an asbestos derivative) degraded with prolonged exposure to a vacuum due to moisture loss. To solve this problem, the motor modules were refitted with ceramic brakes. Future space robotic systems that are exposed to long term vacuum conditions should consider the use of ceramic brake material.

\section{B. Software-Singularity Management}

The flight software (FSW) uses the Jacobian transformation matrix to relate joint rates to rates of the selected control point. When the SRMS is commanded to translate and/or rotate a defined point (tip of the SRMS or a selected point within the payload), the commanded joint rates are obtained using the inverse of the Jacobian. No solution for the commanded joint rates is available when the determinant of the Jacobian matrix is zero. There are three conditions that cause this for the SRMS; these three conditions are shown in Figure 11.

These singularities cause loss of one or more controlled degrees of freedom of the arm. With the wrist yaw joint above the shoulder joints, $\pm \mathrm{Y}$ translation commands cannot be accomplished (shoulder singularity). With the arm straight out, commands in the $+\mathrm{X}$ direction in the EE mode are not possible (elbow singularity). With the wrist yaw joint 90 degrees from the arm pitch plane, some roll commands are not possible (wrist singularity).

These singularities are managed within the RMS software algorithms. The arm is driven through the shoulder and wrist singularities by the software in the automatic and manual modes. During software handling, the arm may deviate slightly from its commanded trajectory. The most dramatic deviation occurs if the operator commands a Y translation through the shoulder singularity. When the wrist yaw joint is 3 feet from being directly over the shoulder joint, the arm is re-oriented to drive in a circle around the singularity and then continues the $\mathrm{Y}$ translation.

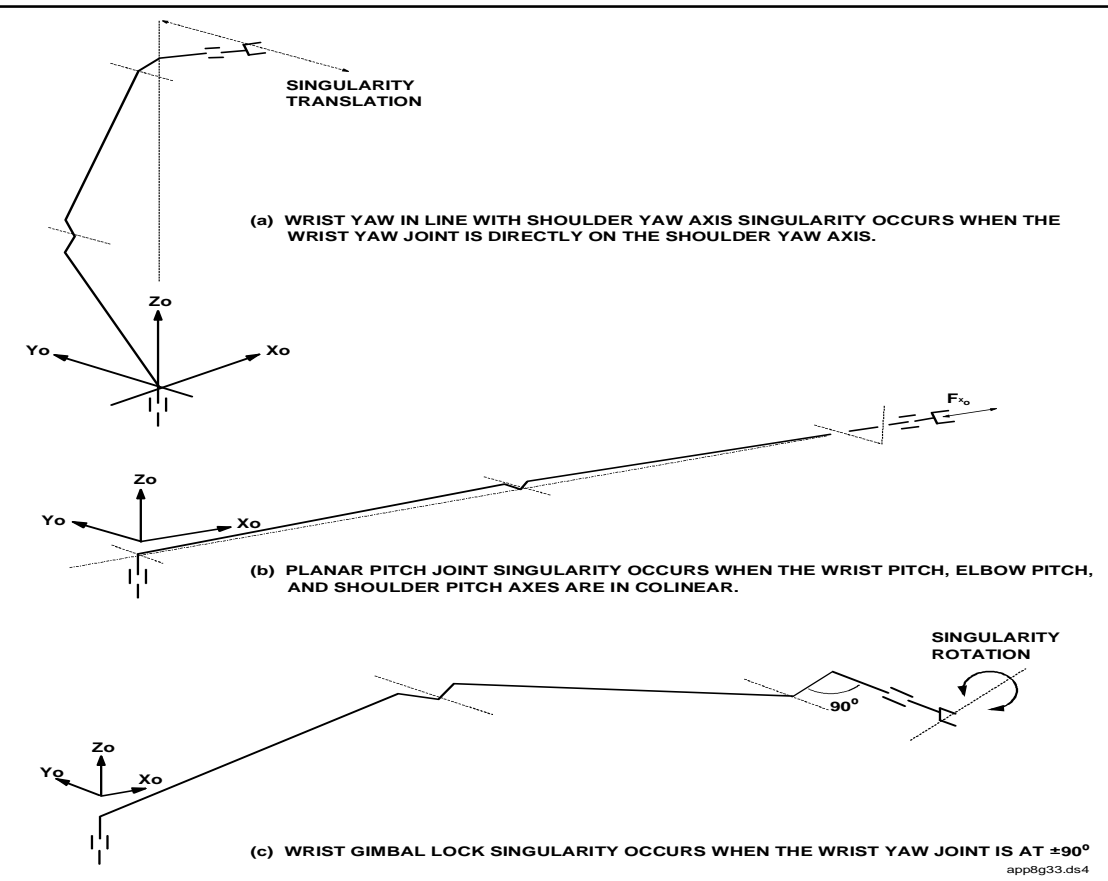

Figure 11: RMS singularity configurations. (Source - Reference 4)

The operator is advised via a caution light on the D\&C panel when a singularity is approached or encountered. The elbow singularity occurs when the SRMS elbow is reaching its maximum reach; motion is permitted until the elbow approaches its limits of motion. This is handled as is any joint reaching its limit. The approach to the limit is first announced by a caution light on the D\&C panel, and then the arm motion is stopped if the operator continues to move further toward the limit. 


\section{Software - Consistency Check}

The SRMS, like most computer controlled systems, uses Built In Test Equipment (BITE) throughout the system to detect errors. In addition, there is also error detection in the FSW, including the consistency check used for runaway detection. Runaways were a particular concern for the SRMS because of the potential for damage to the orbiter if a payload were driven into the orbiter by a joint that had unlimited current applied to the motor. This never occurred during the history of the SRMS, but the potential damage required extra protection to

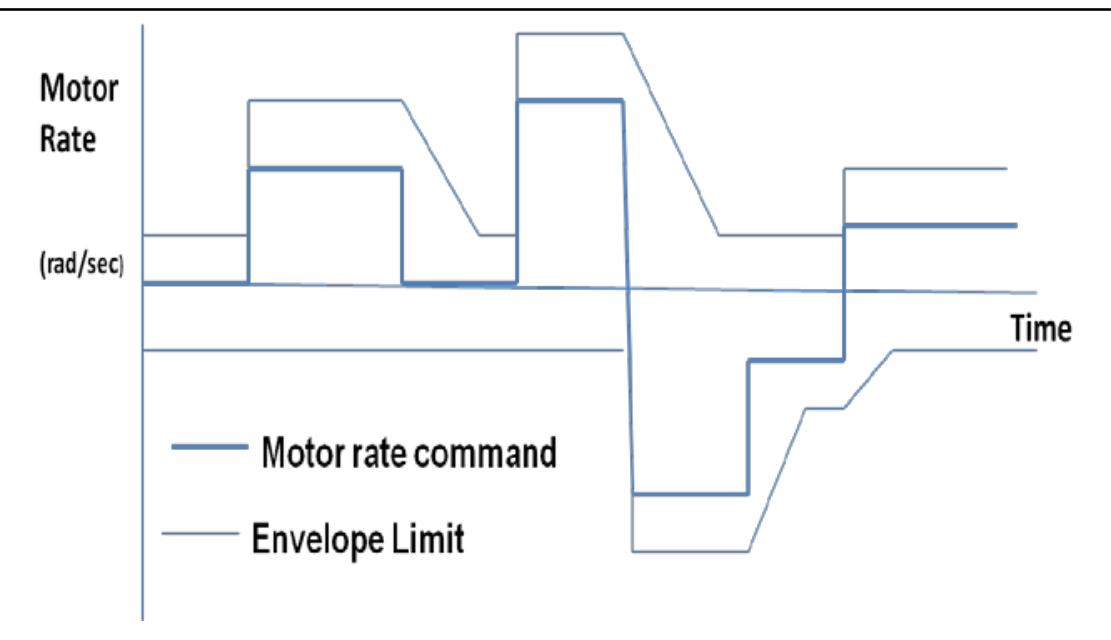

Figure 12: Envelope consistency check for motor rate.

limit the time that a runaway could exist. The consistency check provides an envelope around the commanded motor rate for each joint, as shown in Figure 12.

Three components of the envelope define an offset from zero commanded motor rate, a second offset from a commanded rate, and a decay applied when a command is removed or changed. The actual motor rate is monitored and must remain within this envelope, although dynamic excursions outside the envelope are not annunciated unless the motor rate is outside the envelope for more than 4 consecutive computation cycles. Definition of the values for the envelope constants required considerable analysis at the start of the program to stop joint runaways within an acceptable distance of two feet, while ensuring false alarms were not generated. False alarms could be generated by dynamic excursions in motor rate during command changes.

\section{Test and Verification}

\section{D.1 Air Bearing Test Rig}

Since the SRMS could not support its own weight in a $1 \mathrm{G}$ environment, a unique test method had to be developed to verify the SRMS system as a whole. Individual joint drive tests could be performed with the arm supported in a mechanical "strong-back" ; however testing a complete arm assembly with multi-joint point of resolution control was not possible in this configuration.

An air-bearing flat floor test rig was designed to allow multi-joint operation of the SRMS (see Figure 13). The air-bearing rig allowed forced air to be piped through special air pads mouned on the feet. This counteracted the effects of gravity and reduced the friction between the floor and the test rig loaded with the mechanical arm, allowing the arm to be driven across the floor with minimum loading.

The arm could be mounted on the rig in pitch coupled mode or yaw coupled mode. In pitch coupled mode the three pitch joints could be driven, and in yaw coupled mode the two yaw joints could be driven. The wrist roll joint could be driven in either mode. Even though only one plane of motion could be tested at a time, it did provide engineers a method of verifying arm controls, tip accuracy, tip force, and multi-joint straight line controllability. This was the most comprehensive full up system test performed before the system was checked out on-orbit. Electrical Ground Support Equipment (EGSE) was used to provide power, GPC communication, and data monitoring.

In addition to multi-joint drive, the air bearing test rig provided a means to monitor arm response in a constrained motion envirionment. With the End Effector mounted to the arm, it could be used to grapple a "payload" weighted down to emulate constrained motion (see Figure 13). This configuration was also used to verify the force capability, joint current limit response, and arm response to End Effector/grapple fixture misalignments. 

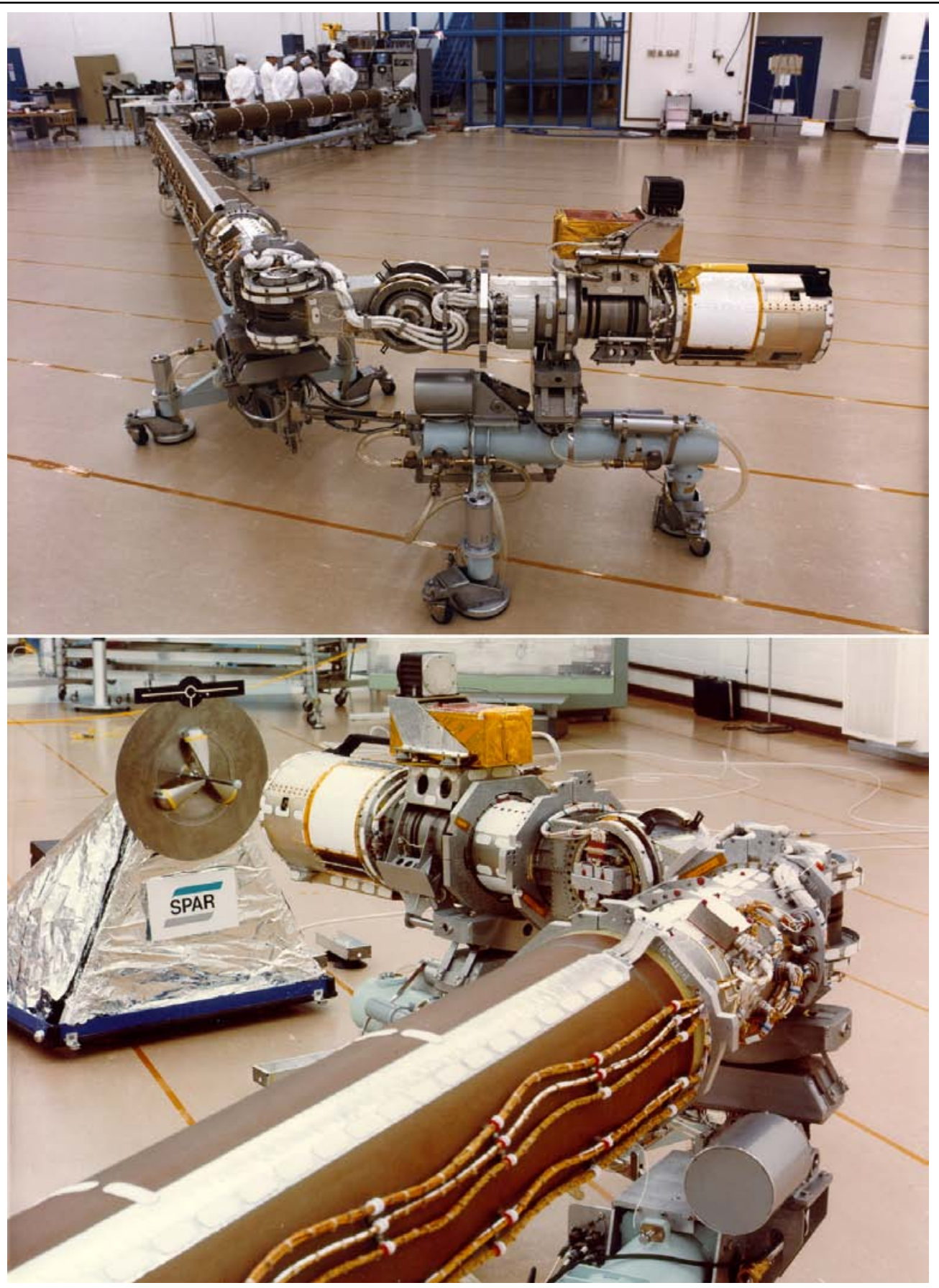

Figure 13: The SRMS on the Air Bearing Flat-Floor Test Rig (Source- Reference 1)

\section{D.2 Simulations}

Simulation of the SRMS was both a solution to the inability to fully test the SRMS on the ground and also presented unique challenges on its own, particularly for real-time simulation in the computers of the 70's and early 80's. When the SRMS was developed, modeling the 6-joint robotic arm was new. The coupled elements ranged from the orbiter (about 6216 slugs) to the light wrist links (the wrist roll joint when no payload was grappled is 3.09 slugs). 
The bending modes of the long booms were also included, with very small effective mass. At JSC the equations were expressed in matrix form and generated a very ill-conditioned matrix. For non-real-time simulations these equations were solved in 64-bit precision. However real-time computers of the day that were fast enough for the SRMS equations generally had only 32 bit precision (and required assembly language programming). This became a significant implementation challenge at JSC.

A major JSC simulation capability throughout the Shuttle Program was the Systems Engineering Simulator (SES), a real-time, operator in the loop simulator that used functional FSW and a functional cockpit. As computers became more powerful throughout the 80's and 90's, the SRMS model was upgraded until it had the full capabilities of the best non-real-time model in 2004.

The models developed for the SES in the 80's and early '90s were also used in the Shuttle Avionics Integration Laboratory (SAIL) which had IBM General Purpose Computers with HAL flight software and a Flight cockpit with the hardware-in-the-loop MCIU for the SRMS. The remainder of the SRMS was a computer model. SAIL, which carried a Shuttle designation (OV-095), was used for verification of the FSW. Connecting the simulated SRMS model to the flight hardware was an additional challenge, involving special purpose hardware boxes as well as careful timing of the SRMS model.

The SES combines the SRMS simulation with 6 DOF dynamic motions of two vehicles. These vehicles are subjected to the effects of orbital dynamics, aerodynamics, the Orbiter Digital Auto Pilot (DAP) thruster firings, the payload control system, and the RCS plume impingement. In the late 90's, in response to the requirements of SRMS berthing studies for ISS assembly, various contact mechanism math models were also incorporated into the SES. The SES also provides the operator with out the window and camera views generated by electronic scene generators. Although scene

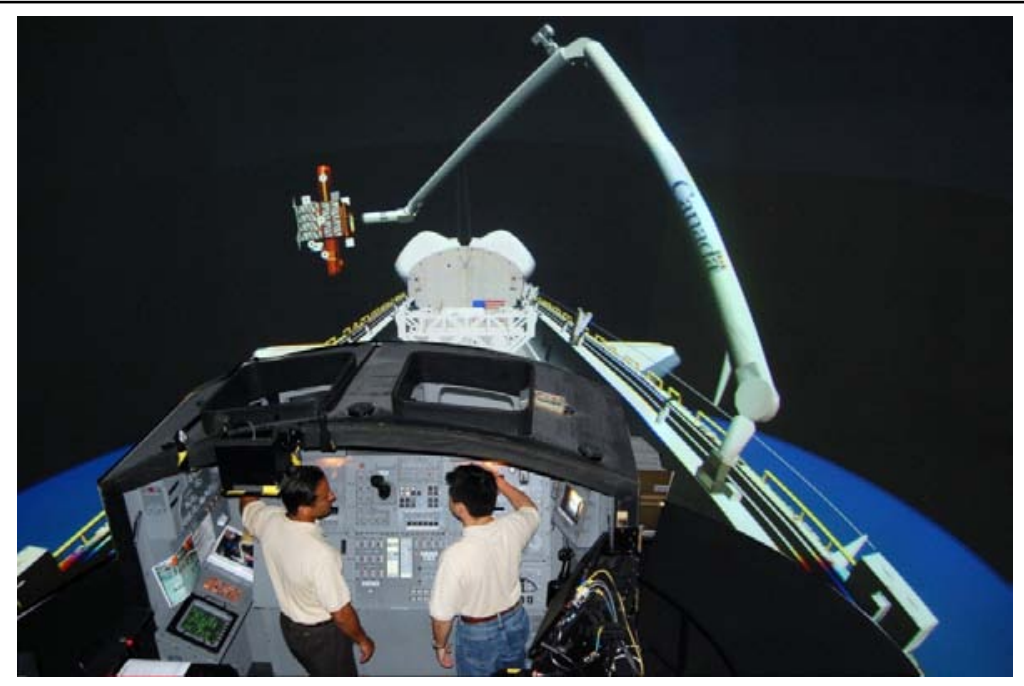

Figure 14. Astronauts Joseph Acaba (at the DAP station) and Akihiko Hoshide (at the SRMS station) in the SES Dome Aft Cockpit with the OTW scene projected on the Dome surface (SourceReference 2) generators are common today, the first electronic scene generator was developed for SES SRMS simulations. The capability was upgraded throughout the Shuttle program and today includes a dome simulator that provides a continuous view from overhead to the Orbiter payload bay. An overview of the SES aft cockpit mockup in the dome display system is shown in Figure 14. Modeling of the DAP as well as the SRMS in the SES allows coordinated operations of the SRMS operator and the DAP pilot. Throughout the Shuttle program the SES was used for engineering evaluations of changes to FSW or procedures that required operator inputs. In addition it was available for procedures development and crew training, and was used many times during flights to evaluate proposed solutions to on-orbit problems. Use of SES included evaluation of procedures for Detailed Test Objectives (DTOs) for the first five flights of the SRMS, track and capture of the first payload released and retrieved by the SRMS (SPAS-01 on STS-7), capture of rotating payloads, and assessing un-commanded motion. Other uses were berthing of payloads to various latching mechanisms on the shuttle and space station, assessment of proposed Orbiter repair techniques, and interaction between various modes of the Orbiter DAP and the SRMS holding the OBSS. It was also used for testing the Position Orientation Hold Submode (POHS) software implemented to control the un-commanded motion.

\section{D.3 Early Missions}

The first five flights of the SRMS included Detailed Test Objectives (DTOs) that started with the simple SRMS capabilities and gradually demonstrated more complex capabilities. The testing conducted on these five flights included: 
STS-2: Unloaded arm tests, including single joint commanding, translational and rotational commanding of the tip of the SRMS, unloaded maneuvers for simulation validation, and auto mode checkout.

STS-3: Additional unloaded arm tests including test of the backup mode, soft stop tests, and PRCS/SRMS interaction. First grapple of a berthed payload, maneuvers around the payload bay, and re-berth. PRCS/SRMS interaction with the payload on the SRMS. A capture of a second payload was not attempted because the wrist camera failed and payload bay cameras did not provide adequate views for re-berthing.

STS-4: Grapple of a payload in the payload bay and maneuver around the bay to assess the payload bay environment, singularity management tests, PRCS/SRMS interaction with an unloaded SRMS and with the payload on the SRMS.

STS-7: Release and capture of a light-weight payload (SPAS-01). Multiple releases in different EE modes were performed to evaluate residual rates imparted on the payload by the release. Track and capture was performed with the arm in manual mode and also single mode. Maneuvers were also performed to provide data for simulation validation.

STS-8: Grapple of a medium weight payload (Payload Flight Test Article) and extensive maneuvering around the payload bay to provide data for SRMS capability checkout and simulation validation. Two grapple fixtures were used. One simulated the inertia of an $8 \mathrm{~K}$ payload and the other simulated the inertia of a $16 \mathrm{~K}$ payload.

\section{Lessons Learned}

\section{A. Off Nominal Operations:}

The primary mission of the SRMS was to deploy and retrieve payloads; however one of the key lessons learned during the life of the Shuttle program is how useful a robotic arm can be for off-nominal and contingency operations. As the missions and additional hardware developed, unique uses of the arm emerged. Over the life of the program, the SRMS allowed the mission management team to deal with a variety of unforeseen problems encountered on orbit. The reach and force capability of the SRMS provided an operational flexibility that was not available with any other shuttle system. A robotic arm provides an effective solution for many tasks that formerly either could not be performed or required EVA to perform. Some unique operations performed were applying force to a satellite antenna to lock it in place (see Figure 15), supporting an EVA crewman performing a repair on Thermal Protection System of the Shuttle (see Figure 16), "ice busting" on STS-

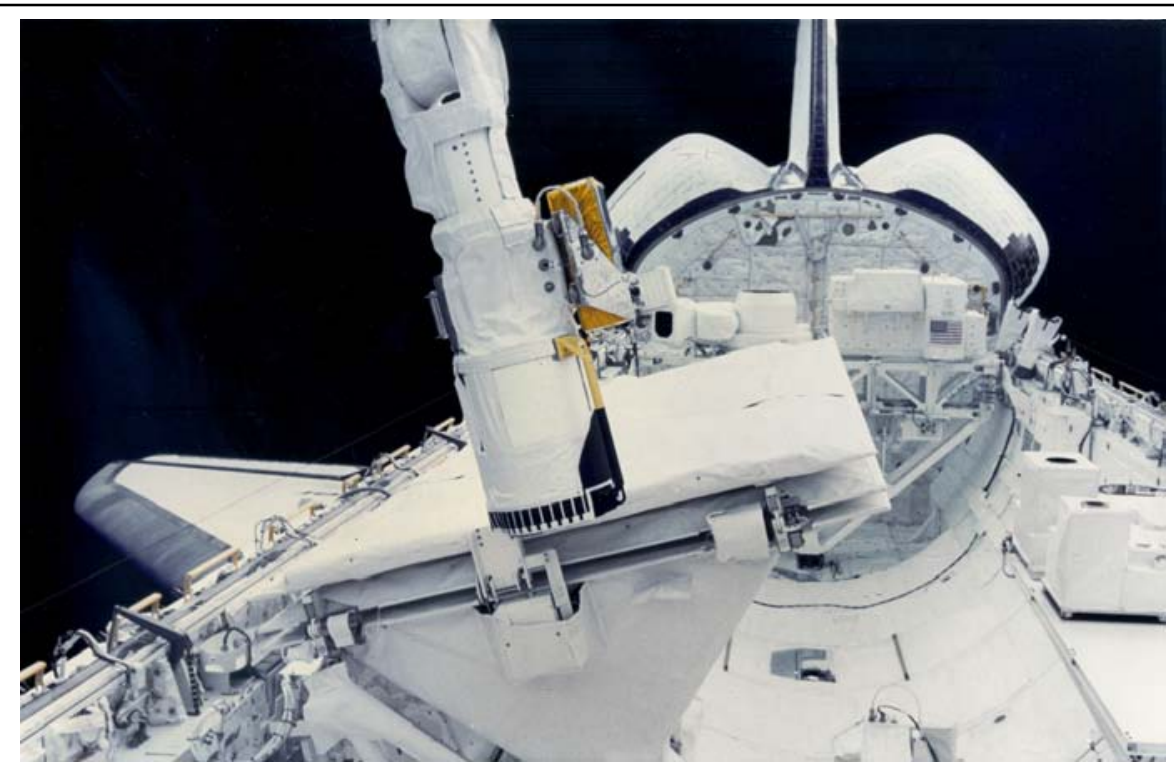

Figure 15: STS-41G Latching Down the SIR-B Antenna (Source- Reference 1) $41 \mathrm{D}$ to remove a large icicle that formed on the shuttle's waste nozzle; and "fly swatting" on STS-51D to engage a switch lever on a satellite that had been incorrectly positioned. 


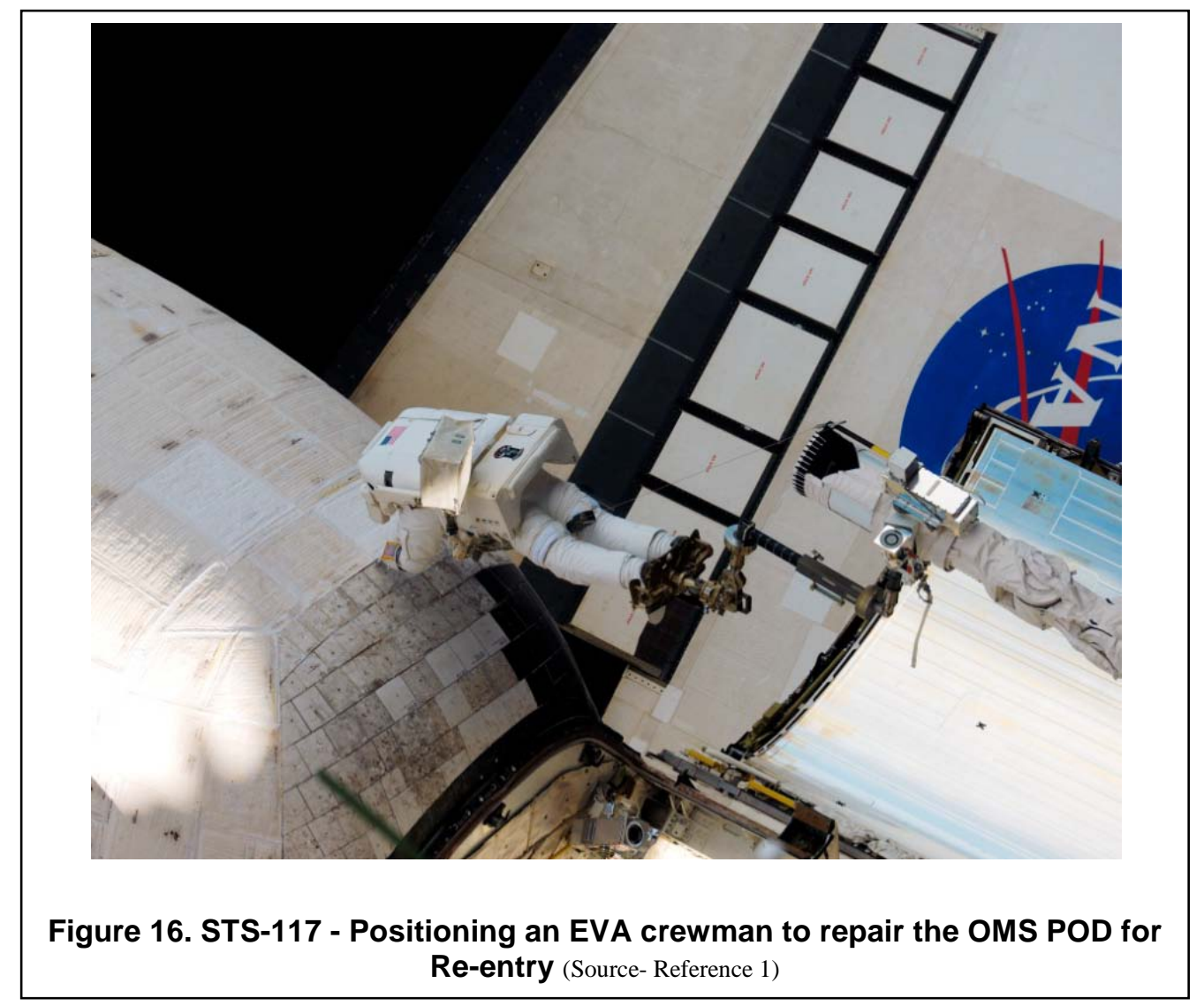

\section{B. Position Orientation Hold Sub-mode:}

A key lesson learned during the SRMS program was the improvement in arm performance that could be achieved with the addition of Position Orientation Hold Sub-mode (POHS) to control un-commanded motion. During STS-8 while the SRMS operator was maneuvering the PFTA payload, he noted that many corrections were needed to control un-commanded motion. During the LDEF un-berthing on STS-41C, crew started the un-berthing with a pure z command, as shown in Figure 17. This provided very clear data on un-commanded motion. As seen in the figure, the only command is a THC z-command, but at about 11.5 inches of $\mathrm{z}$ translation, x moved about 2.5 inches (about 22\% of the motion in the commanded direction), roll moved about 1 deg, pitch moved about $1 / 2$ deg (after first moving a small amount in the opposite direction), and the other axes moved by smaller amounts. Un-commanded POR motion is caused by a number of factors including torque disturbances due to dynamic coupling between the joint, friction, etc. In particular, when any joint rate crossed zero and friction torque changed sign, the un-commanded motion increases. This un-commanded motion was more significant when the commanded rate is low, and it became particularly objectionable for the deployment of heavy telescopes in the early 90's. During the HST deployment on STS-31 in April 1990, crew commented on the heavy workload due to the un-commanded motion. Figure18 shows the THC commands and the resulting translational motion, and Figure 19 shows the same data for the rotational axes. The unberthing command is a pure $\mathrm{z}$, but that command stops and starts and many commands are also seen in all other axes. 


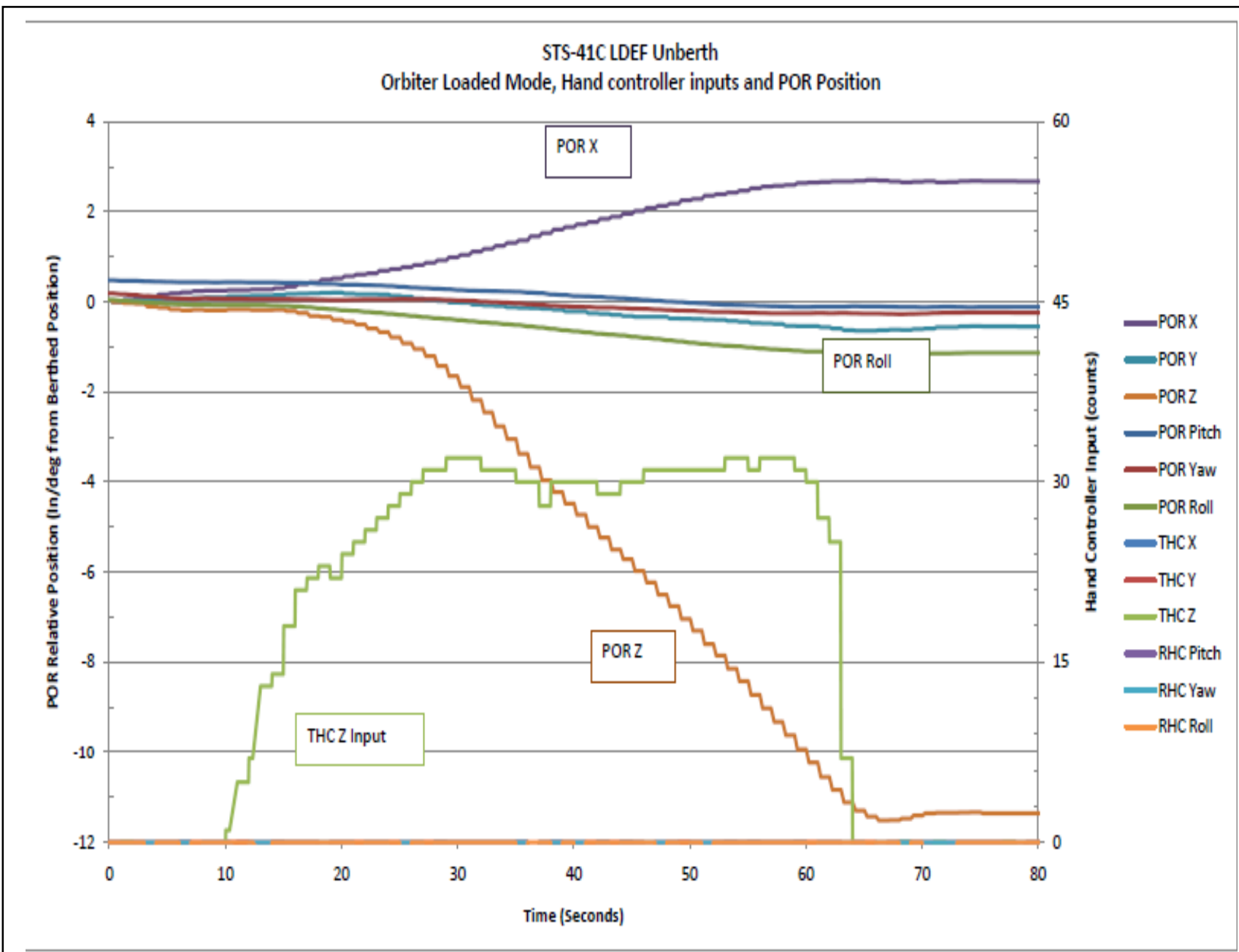

Figure 17. POR commands and POR actual motion during the first part of LDEF unberthing on STS-41C 


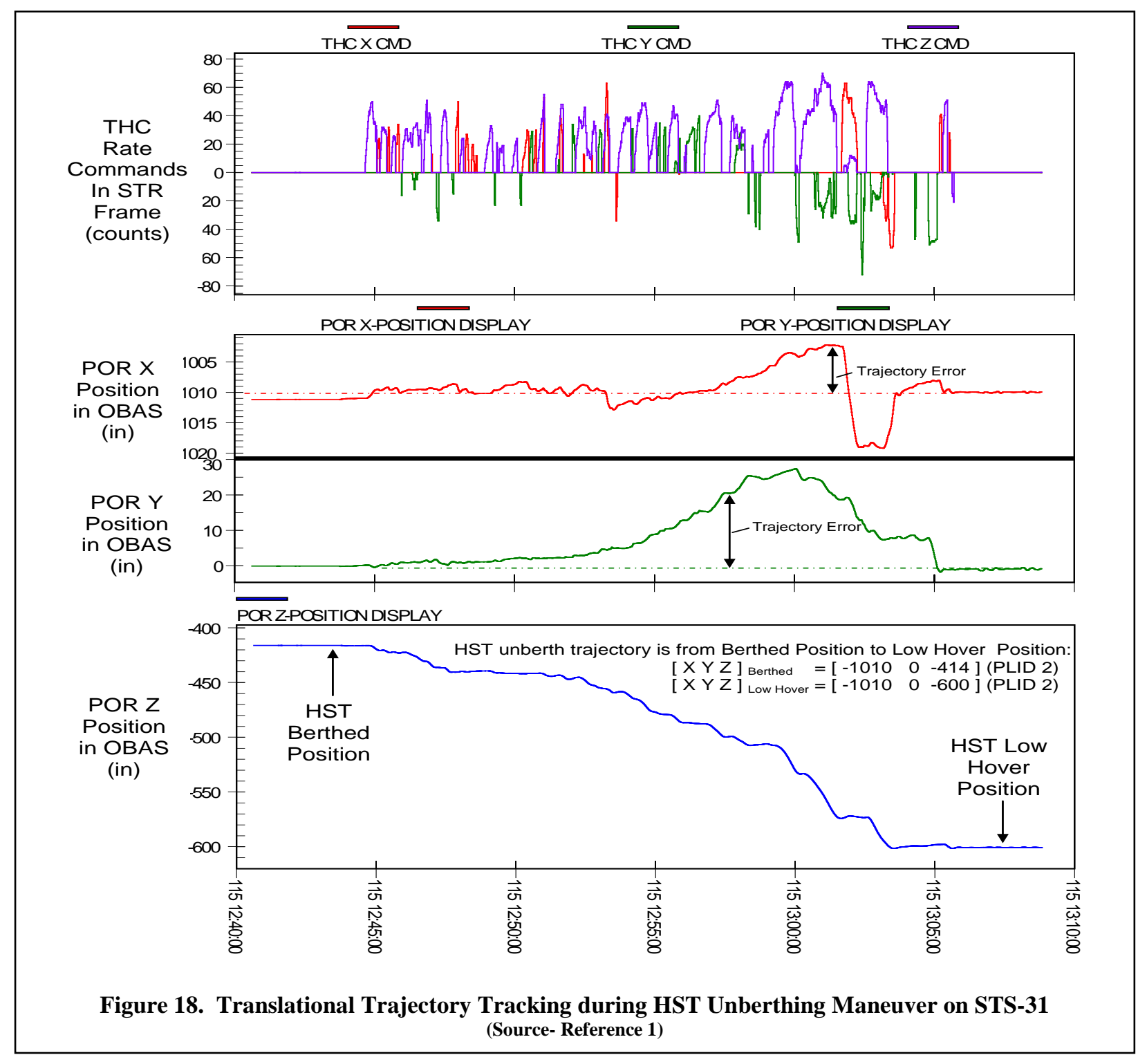




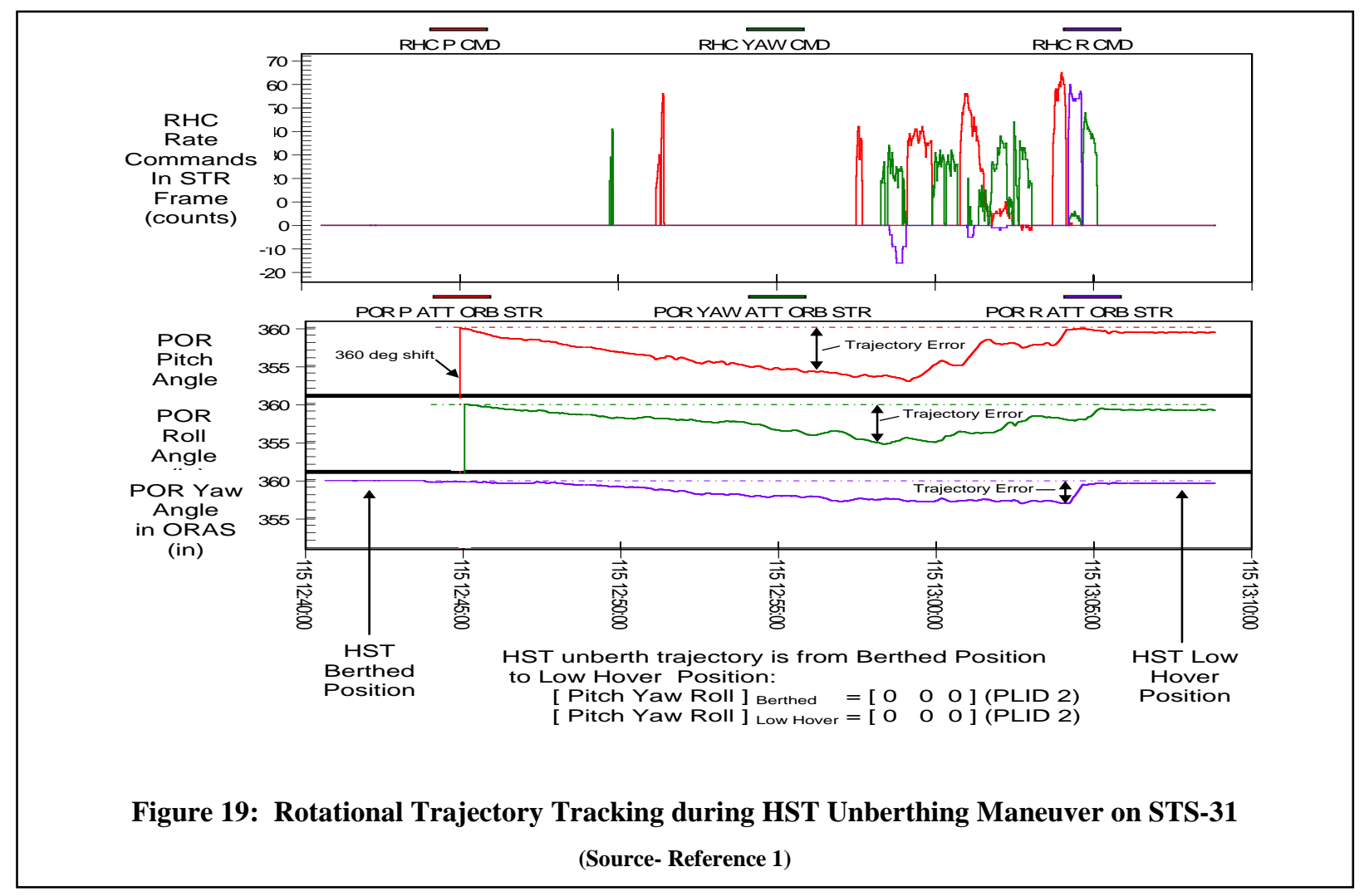

To address this issue, POHS software was developed. The SRMS is a rate command system, and prior to the implementation of the POHS software, POR position and attitude were computed for display only. The POHS software uses the POR position and the commanded rate as shown in Figure 20. The figure shows two periods of rate command with a period of null command between them. When the SSRMS is at the initial POR position, the operator puts in the command $\mathrm{V}_{1}$. The POHS software projects a reference trajectory and at each software update cycle (80 msce) compares the actual POR position to the projected position at that same time. The error perpendicular to the reference trajectory is used to generate POHS commands that will bring the actual position back to the projected position. When the operator command is nulled, the reference trajectory is projected based on the last command until the rates drop below a defined threshold value. At that point POHS goes into position hold and commands are generated

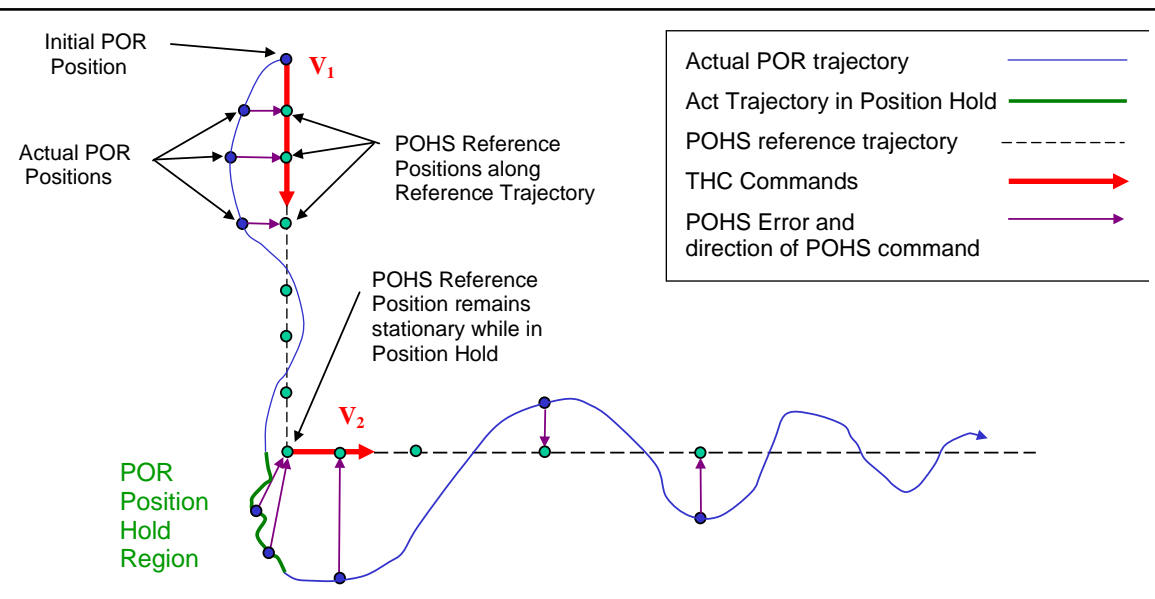

Figure 20: POHS Control of POR Trajectory

(Source- Reference 1) 
to return the SRMS to that point if any un-commanded motion occurs. Figure 20 shows one axis of the POR computation for position; for attitude, the POHS software uses a total angle deviation from the projected attitude to generate the POHS commands.

POHS software was available for the HST servicing missions after STS-64, and the reduction in workload was very striking during HST unberthing operations. During the missions the HST is unberthed when it is vertical in the payload bay and it was horizontal in the bay during the STS-31 unberthing, but the unbething rates were similar for both unberthings and commanded rate is the major driver for uncommanded motion. Figures 21 and 22 show the hand controller commands and actual POR motion for the STS-103 HST unberthing. These figures show deviations of less than 1 inch and 1/2 degree. STS-31 deviations were larger than 25 inches and almost 10 deg. There were also no operator corrections during the STS-103 unberthing with POHS. There was one roll correction before the unberthing started, but no attitude or position corrections during the unberthing.

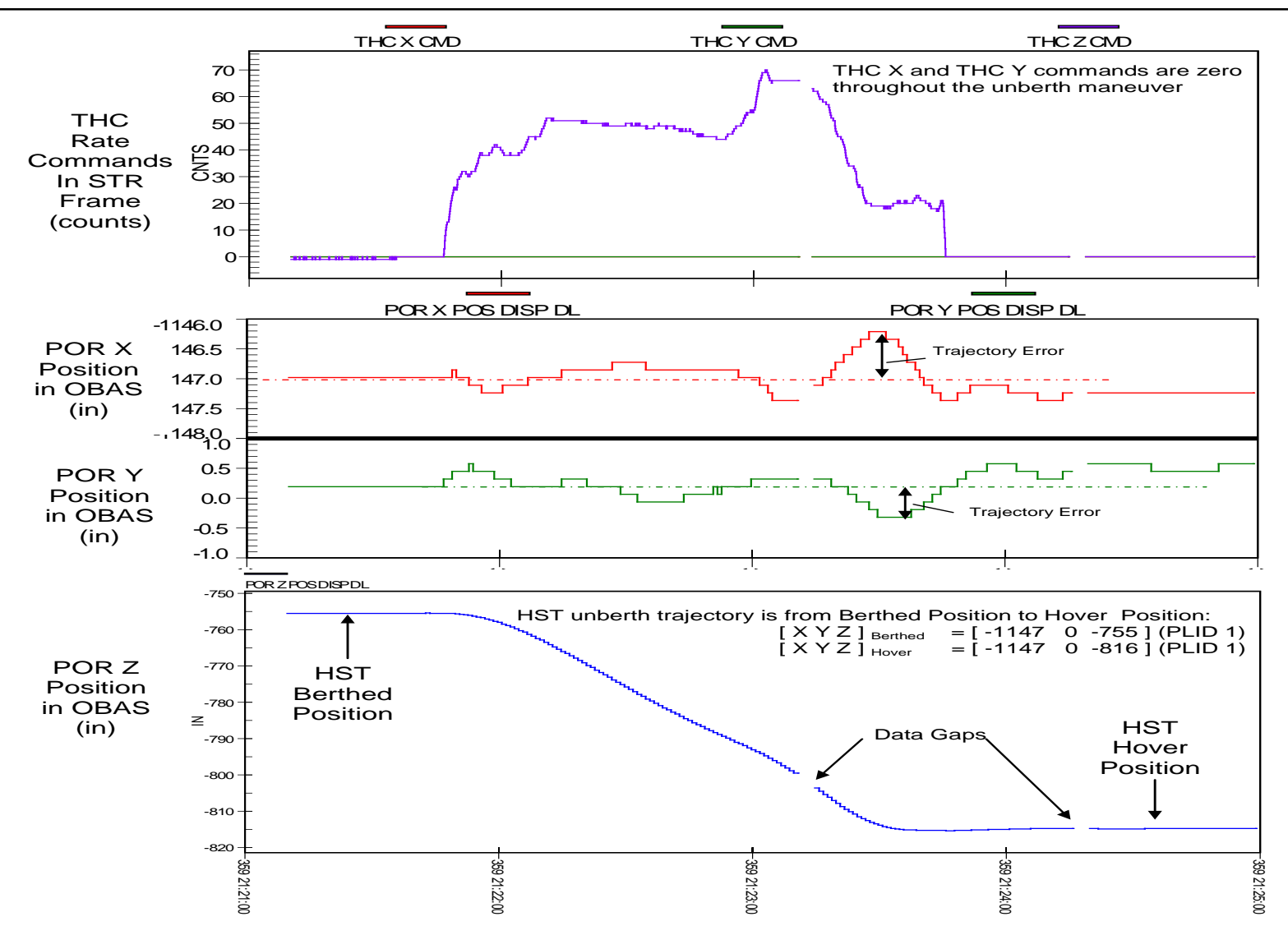

Figure 21: Translational Trajectory Tracking during HST Unberthing Maneuver on STS-103

(Source- Reference 1) 


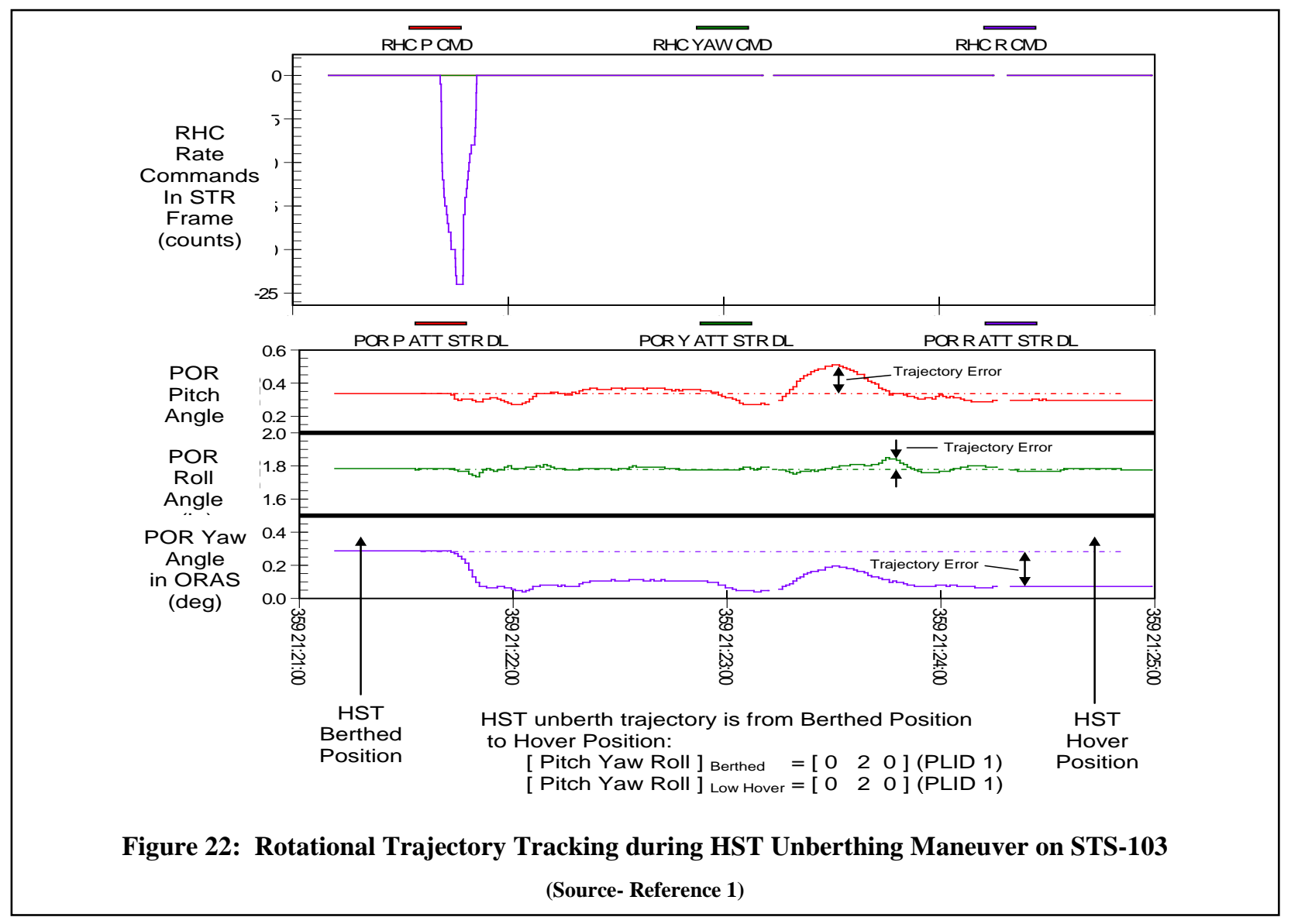

The POHS software was first implemented in Manual Augmented modes to address the operator workload issue. When Auto modes were used for Orbiter tile inspection after the Columbia accident, the operator had limited ability to monitor the OBSS while the boom was under the orbiter. To address this, the POHS controller was added to auto modes. However, an undesirable attitude oscillation was noted in position hold when the sensors on the OBSS scanned a specific point. This was traced to GPC precision for the very small angles seen during position hold, and the algorithm for calculating attitude error was changed to use a function that was less sensitive to the small angles.

The value of POHS was reinforced during ISS assembly operations when the SRMS unberthed heavy modules and truss segments from the shuttle payload bay, often with small clearances between trunnions and the OBSS or other elements in the payload bay..

\section{Command Wash-in in Auto Mode:}

Auto modes and single joint mode both command to a single rate, but they start the commands differently. In single joint mode the commands are ramped up over several computer cycles but in the auto modes the full command is put in instantaneously. After the Columbia accident, wash-in was also proposed for auto modes to improve the transient response, as discussed below. The existing and proposed auto mode command profiles are shown in Figure 23. In both single joint and auto modes the command is washed out as shown in Fig 23, because abrupt nulling of command would cause the flexible SRMS to have significant overshoot and oscillations in POR and POR rate. There are similar overshoots and oscillations with a step input, and these were
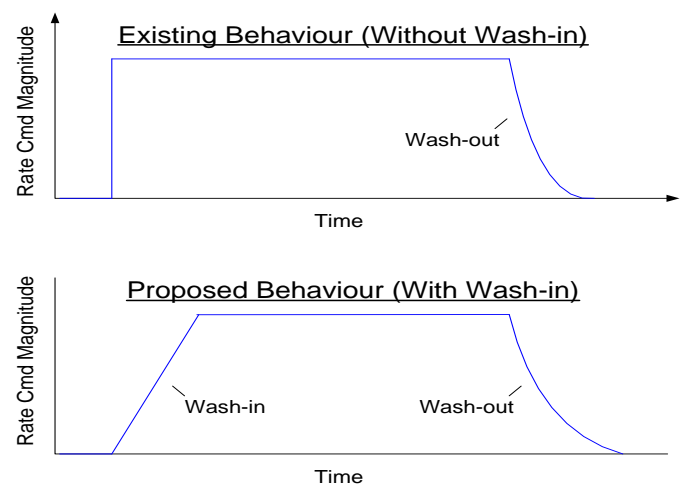

Figure 23: Comparison of Existing and Proposed Wash-in Rate Command Profiles for Auto Mode

(Source- Reference 1) 
sufficiently objectionable in single joint mode to force wash-in of commands. For auto modes, the commands were initially started far enough from structure that the oscillations were not objectionable.

When auto modes were used for OBSS inspections, the sensors had rate limits above which the images were not adequate for clearing tile for entry. The rate overshoots then drove reductions in the command rates to meet sensor requirements. Wash-in of the auto mode was proposed at this time, but it was decided that the reduction of command rate was an adequate solution. The effect of wash-in for a TPS inspection auto sequence is shown in Figure 24, which compares the rates at the start of the same auto mode with and without wash-in. The sensors had requirements to find 0.25 inch and 1.00 inch damage at different parts of the survey. For 0.25 in damage the existing software requires a POR translation rate of 0.5 meter/minute and with wash-in the rate could have been increased to 0.6 meter/minute. For 1 inch damage, the translation rate must be 1.4 meter/minute with the step input; with wash-in the translational rate limit could have been 2.7 meter/minute.

While wash-in was not considered necessary for the SRMS, it is an improvement that should be considered seriously for any future space robotics.

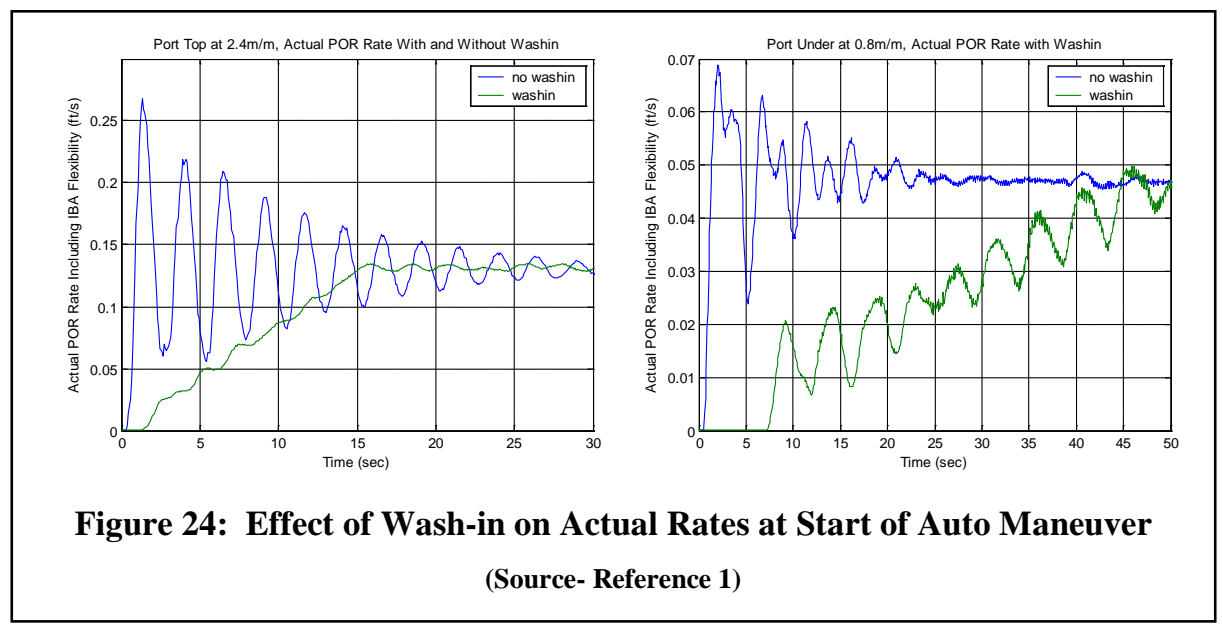

\section{Value of Open Review of Simulation Models:}

At the start of the SRMS program, several different implementations of the SRMS equations of motion were developed by organizations including Spar, Rockwell Downey, JSC, and CS Draper Labs. At the time modeling a robotic space-based robotic system with the many degrees of freedom of the SRMS was a significant technical challenge for each organization. Early in the program, the model developed by the hardware provider Spar was designated as the "truth" model by the Program Office; however, the simulation was considered proprietary and the model and implementation were not disclosed. The truth sim, and all other sims, did not always match well with hardware data and sim responses did not match each other well. These differences between sim responses were a major topic of discussion in SRMS forums for 20 years. Even comparison to flight data did not resolve the issue since the errors from all sims were about equal, particularly for loads. As discussed in the section "Enhanced Instrumentation", this was due to limitations of the flight data.

Little progress was made in resolving model/implementation differences until 1999 when the SRMS Program office insisted on development of a "common" SRMS model and chartered the SRMS Math Model Working Group (MMWG) to coordinate open discussion of all model features and implementation. This resulted in codevelopment of an SRMS model that was accepted as the standard for the remainder of the Shuttle program.

For any system used in a Program, a single model and implementation that is open for review and well grounded in ground testing and flight data should be provided for use throughout the Program

\section{E. Load Relief of Space Based Robotics:}

The SRMS was designed with relatively weak brakes at the wrist, a stronger brake at the elbow, and even stronger brakes at the shoulder. As a result, the wrist brakes slip under high load, and the orbiter does not see high loads at the longeron. The RMS for the ISS (SSRMS) made a different design choice to allow inchworm capability for the 
SSRMS. Either end can be the shoulder, so all joints have high strength brakes. As a result, the SSRMS can transmit a high load to the base, a feature that has required operational workarounds, particularly to avoid potential high loads if the SSRMS brakes come on as a result of a safing event while a berthing mechanism is pulling an element held by the SSRMS. For berthing with the element held by the SRMS, the brake torque scaling avoided this problem.

A potential solution that would allow inchworm capability and also load relief would be use of dual brakes at each of the three end joints, with one set scaled to allow load relief, and a second brake to bring the total torque up to the shoulder value. Positive lockout of the second brakes at the joints that are acting as the wrist must be provided. The elbow joint brake torque could be scaled as the SRMS elbow brake torque was.

Dual brakes on each of the three end joints would add mechanical complexity and weight to the robot; these need to be weighed against the reduction in operational complexity when load relief is important.

\section{F. Enhanced Instrumentation:}

The SRMS electronics provided motor rate and joint position data to the operator, and a later upgrade to the Arm Based Electronics added motor current data that was available for viewing on the ground. The original arm had a set of strain gauges, called Development Flight Instrumentation (DFI), installed however the data was of limited usefulness due to factors such as low resolution, poor calibration and the difficulty of retrieving the data. These limitations were largely due to the state of the technology at the time of development. After the Columbia accident one of the arms was instrumented with strain gauges and a test was performed on orbit with a crewman on the end of the OBSS to simulate a repair scenario. This data was useful, however the test was limited to this one scenario only. No data was ever collected when the arm was maneuvering large payloads, or when the arm was operating in a constrained motion scenario.

As the SRMS was utilized for more varied tasks as the program progressed, including manipulating much larger payloads, it became clear that force and moment data would be very useful. Examples of this include using the OBSS on the SRMS as an EVA platform for repair of the shuttle thermal protection system, and certifying the SRMS to grapple the ISS and maneuver the entire Shuttle. This data would allow engineers to understand the loads the SRMS was exposed to which would aid in life cycle estimates. In addition, loads data would be very valuable for simulation. Several simulators of the SRMS were developed over the life of the program and these simulations played an integral part in certifying SRMS/payload operations. The major difficulty encountered was validating the various models that were developed for the arm. Due to the uncertainty in models developed from analysis only, conservative assumptions were made to ensure the uncertainty was bounded. Proper instrumentation on future robotic systems would aid greatly in the development of accurate models and limit the amount of analysis required. Additionally, significant analysis has been devoted to analyzing situations when loads can buildup due to contact with a berthing mechanism, grapple fixture, etc. This analysis is required because the only times that the SRMS was instrumented, the loads were not recorded when there was contact between the SRMS or payload and hardware attached to the Orbiter. The complexity of modeling contact surfaces has raised many questions that increased the analysis impact. Adding load instrumentation to a future robot would allow validation of contact load models with flight data.

\section{G. Force Moment Accommodation:}

Deploying and retrieving payloads primarily involves maneuvering payloads that are unconstrained. Since this was the mandated task of the SRMS it was designed for pure coordinated joint rate control. However, for constrained motion when the payload can contact external structure, coordinated joint rate control is no longer possible. In this scenario, the direction of the applied tip force/moment will be altered and will not be as commanded by the operator hand controllers. This will result in motion of the arm tip in un-commanded directions. This makes constrained motion activities more difficult for the SRMS operator. Force moment accommodation (FMA) can greatly facilitate SRMS tasks involving constrained motion operations.

The lack of FMA during the construction of the ISS underscored how useful it could be. The SRMS operational requirements expanded to include assembly tasks involving the mating of modules during the ISS construction. For these operations the limitations of velocity control became apparent. Mating operations required force application between surfaces that had to be correctly aligned for proper mating. Since misalignments are inherent in space robotic operations with limited visibility, this alignment uncertainty had to be addressed. Sophisticated visual aids were required for the SRMS operator to place the mating surfaces within a predefined capture envelope, and active 
mating latches were required to provide the force necessary to overcome the remaining misalignments within this envelope. Additionally, extensive preflight analysis of all the potential contact conditions during misalignments was necessary to ensure load limitations of the SRMS were not exceeded.

Force and moment data displayed to the operator would provide enhanced situational awareness thus improving positioning accuracy. The increased positioning accuracy of the payload/mating interface would reduce the reliance on visual cues. The sensed force and moment data implemented in a feedback loop would control contact forces improving constrained motion operations. By controlling contact forces, FMA would allow the SRMS/payload to adapt to uncertainties in the contact environment, safe guarding against damage caused by excessive loads. This minimizes the need for pre-mission loads analysis. With the capability to apply constant, pre determined forces in a given direction, the SRMS can be used to activate berthing and latching devices. Active mating mechanisms would not be necessary, simplifying the mating interface design.

Additionally, FMA can extend the capability of a robotic arm to perform complex and delicate tasks needed for satellite servicing. These tasks include refueling, module change outs, cleaning of surfaces, electrical connector mate/de-mates, and thermal blanket change outs.

\section{H. Expansion Capability:}

The SRMS end effector was designed with an electrical connector called the Special Purpose End Effector (SPEE) connector. This was envisioned as a means of providing power and data to future specially designed end effectors that were grappled by the standard SRMS End Effector. This connector could also electrically mate with a special grapple fixture called an electrical flight grapple fixture (EFGF). Payloads with this grapple fixture could utilize the sixteen power and data lines that ran the length of the arm in conjunction with switching panels in the orbiter that were configured preflight as needed. When the OBSS was developed, it utilized an EFGF to provide power and a data path for the cameras and lasers on the end of the boom. During the design phase it became clear that the existing SPEE cables could not provide the required electrical current needed by the sensors at the Shuttle bus voltage of 28 Volts. The work around was to use a 120 volt power source in the shuttle that was designed for use on the ISS.

The limited capability of the original design of the SPEE wiring harness was a lesson learned for robotics on future space vehicles. Robotic systems of the future should be designed with future growth in mind. While it is difficult to predict future requirements, and cost limitations may restrict the amount of enhancements, it is still prudent to provide additional power and data paths that can be utilized for future capabilities.

\section{Conclusion}

The Shuttle Remote Manipuilator System represents one of the many great legacies of the Space Shuttle. Its unique capabilities were recognized as the program developed, and it evolved into much more than just a means of deploying and retrieving satellites. From an EVA platform, to satellite rescue and repair, to the inspection of the thermal protection system of the shuttle, it met the challenges of ever more complex space missions. Its success was a major driver in developing the Space Station Robotic Manipulator System on board the International Space Station today, and it will serve as the foundation for all future space robotic systems.

\section{References:}

1. MDA-RMS-R.10786 "SRMS Technical History and Lessons Learned”

2. "Wings in Orbit - Scientific and Engineering Legacies of the Space Shuttle" (NASA)

3. Space Shuttle Orbiter Operational Level C Functional Subsystem Software Requirements Remote Manipulator System (RMS)

4. NSTS 07700, Volume XIV “System Description and Design Data- Payload Accommodations”, Appendix 8 "Payload Deployment and Retrieval System Description” 


\section{Acknowledgments}

The authors would like to acknowledge the excellent work McDonald Dettweiller Associates (MDA) has performed in documenting the history of the SRMS program in reference 1.

\section{References}

The following pages are intended to provide examples of the different reference types, as used in the AIAA Style Guide. When using the Word version of this template to enter references, select the "references" style from the drop-down style menu to automatically format your references. If you are using a print or PDF version of this document, all references should be in 9-point font, with reference numbers inserted in superscript immediately before the corresponding reference. You are not required to indicate the type of reference; different types are shown here for illustrative purposes only. 


\title{
Shuttle Remote Manipulator System History, Evolution and Lessons Learned
}

\author{
Elizabeth Bains \\ Glenn Jorgensen
}




\section{Outline}

- SRMS Overview

- SRMS History

- Unique challenges

- Lessons Learned 


\section{SRMS Overview}

\section{Requirement}

- Deploy and retrieve satellites from payload bay

- 50 foot long anthropomorphic arm

- Teleoperated from aft crew cabin

\section{Manipulator Arm}

- 3 joints - 6 DOF

- 2 arm booms

- End Effector

- Three camera assemblies

- Thermal protection system

Cabin Equipment

- Displays and Controls panel with rotational and translational hand controllers

- Manipulator Controller Interface Unit interface between Shuttle GPC, D\&C

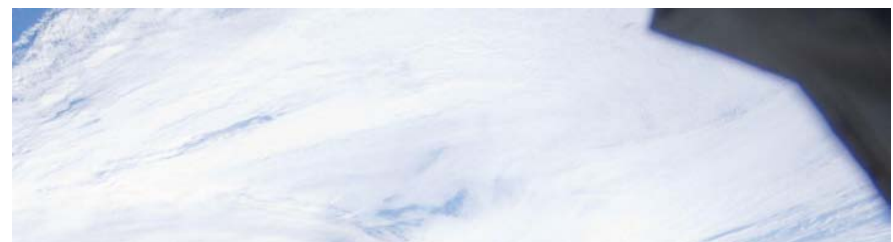
panel, and Manipulator Arm electronics

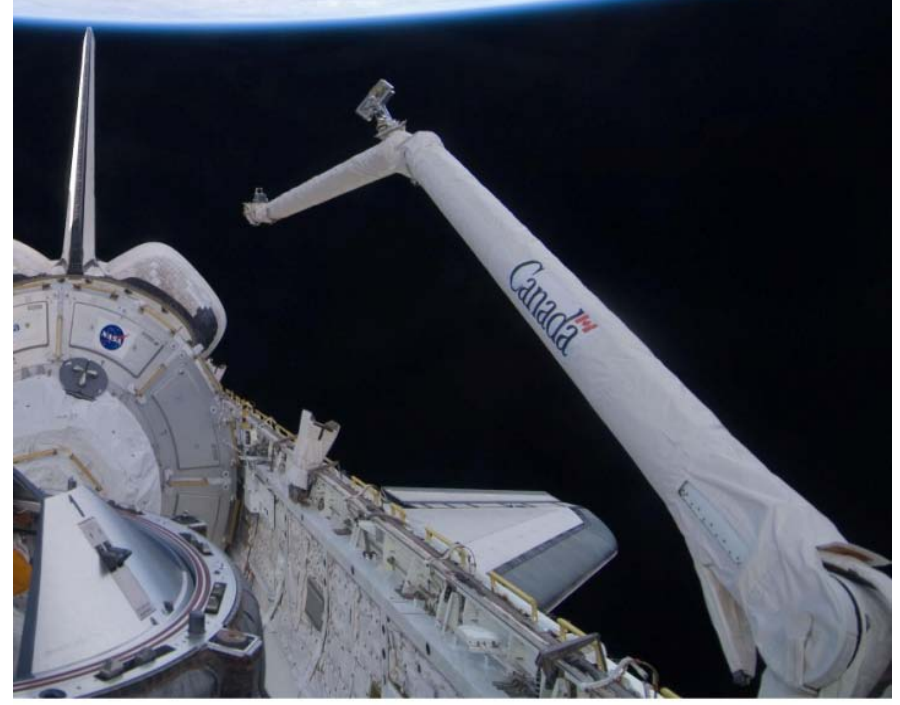




\section{SRMS Overview}

Translational Rotational Handcontroller Handcontroller

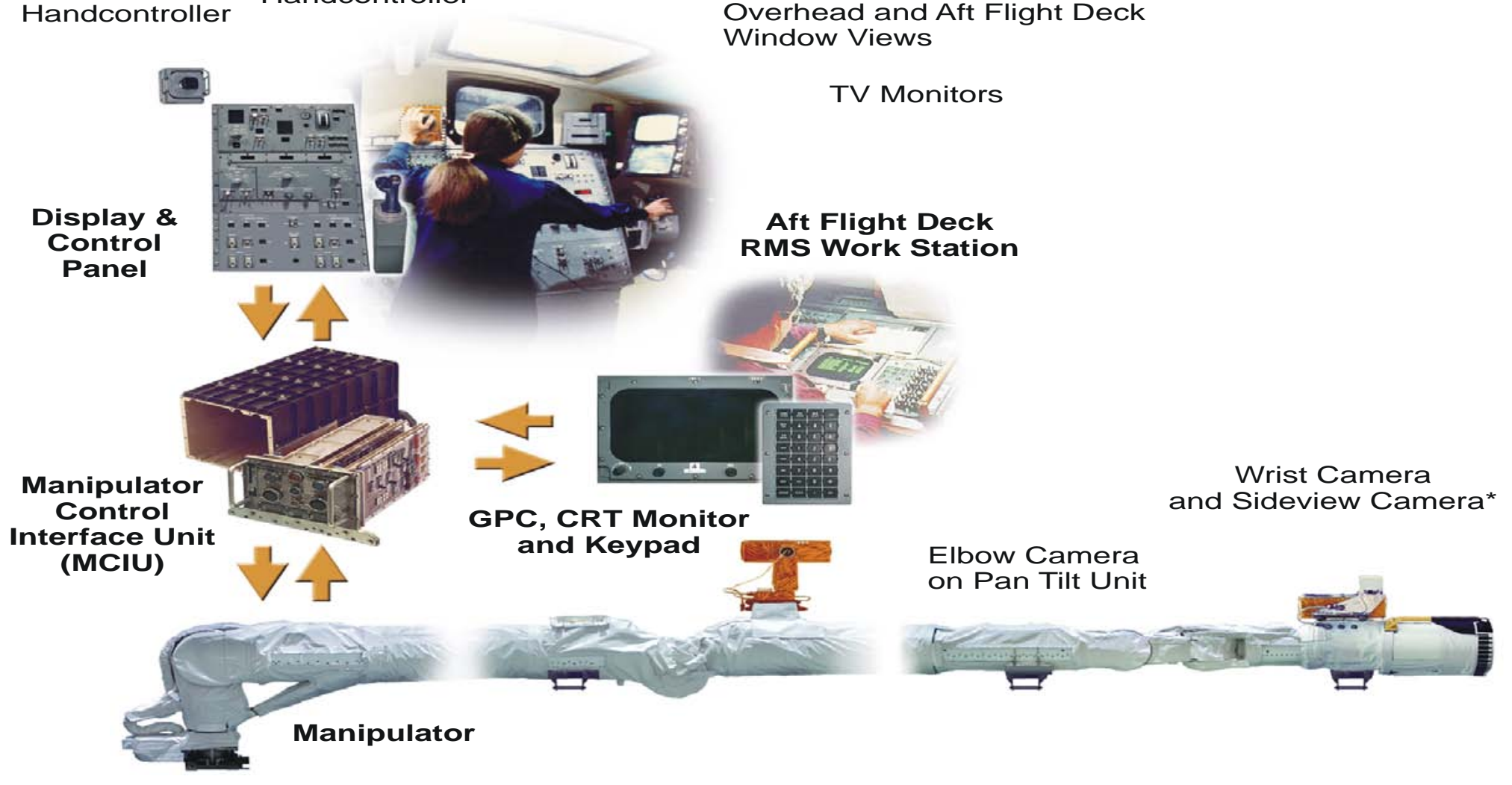

\section{SRMS Components}




\section{SRMS Overview}

\section{SRMS End Effector}

- 3-Cable mechanism snares a grapple fixture on payload

- Snare mechanism is retracted to "rigidize" payload to End Effector

- Grapple target used with Wrist camera
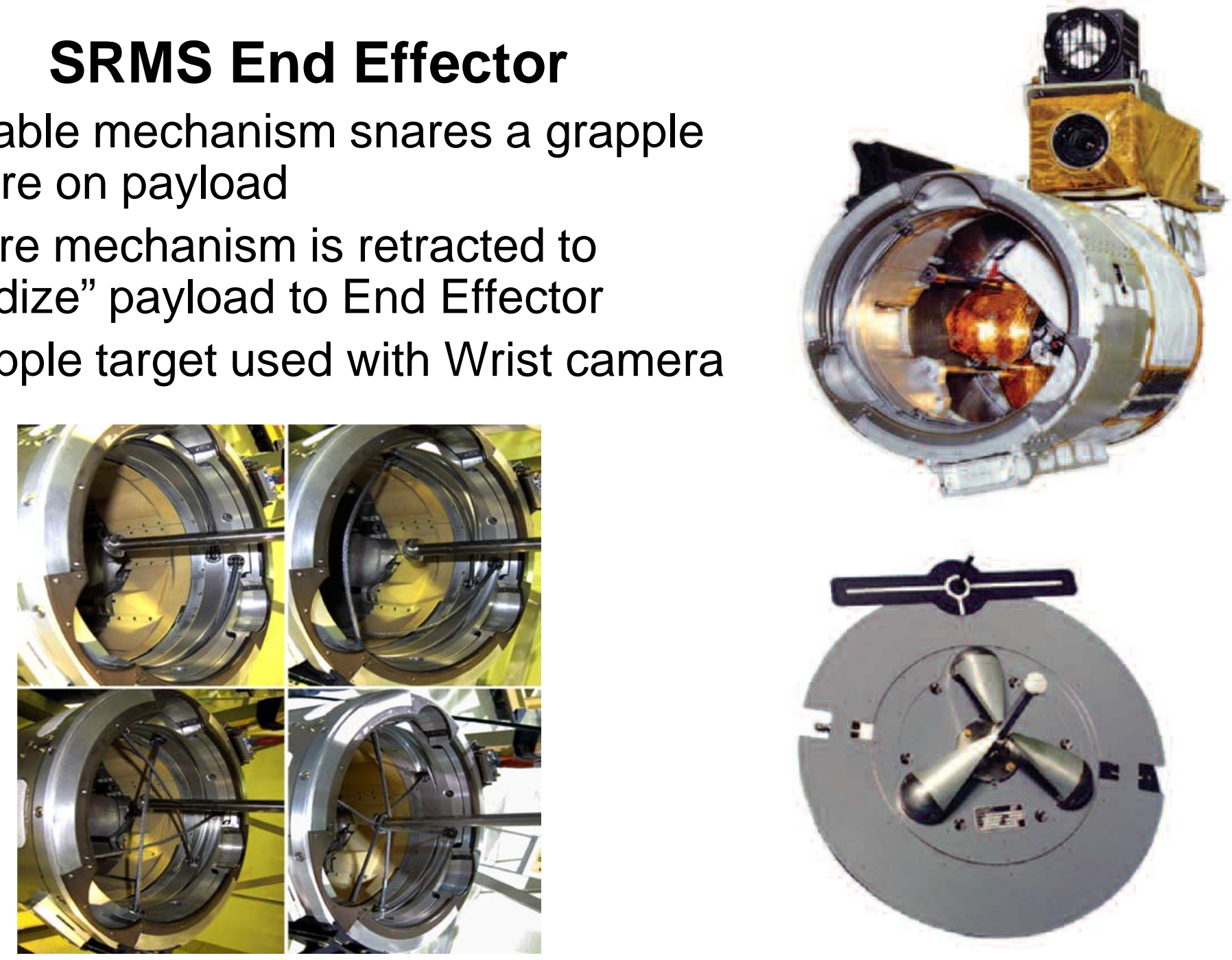


\section{SRMS Overview}

\section{SRMS Operating Modes}

- Primary Modes

- Computer Supported Modes

- Coordinated servo rate control - rates are pre-programmed

- Automatic Sequences - Pre-programmed and Operator commanded

- Manual Augmented Modes

- Point of Resolution control via RHC and THC commands

- POR and Co-ordinate axes based on one of five modes chosen by operator

- Payload POR determined pre-flight

- Single Drive

- Single Joint Driven - other joints in position hold

- Direct Drive Mode

- Single joint driven with a fixed voltage - no servo rate control

- Back-Up Drive

- Separate electronic drive

- Single joint driven with a fixed voltage - no servo rate control 


\section{SRMS History}

\section{Satellite Deployment}

- Gamma Ray Observatory (GRO) on STS-37

- Earth Radiation Budget Satellite (ERBS) on STS-41

- Upper Atmosphere Research Satellite (UARS) on STS-48.

- Satellite Deployment and Retrieval

- SPAS-1 satellite on STS-7 -first free flyer capture

- Long Duration Exposure Facility (LDEF) deployed on STS-41C, and retrieved on STS32

- Solar Maximum Mission satellite (Solar Max) on STS-41C

- Hubble Space Telescope (HST) - deployed on STS-31, retrieved on 4 missions for repair
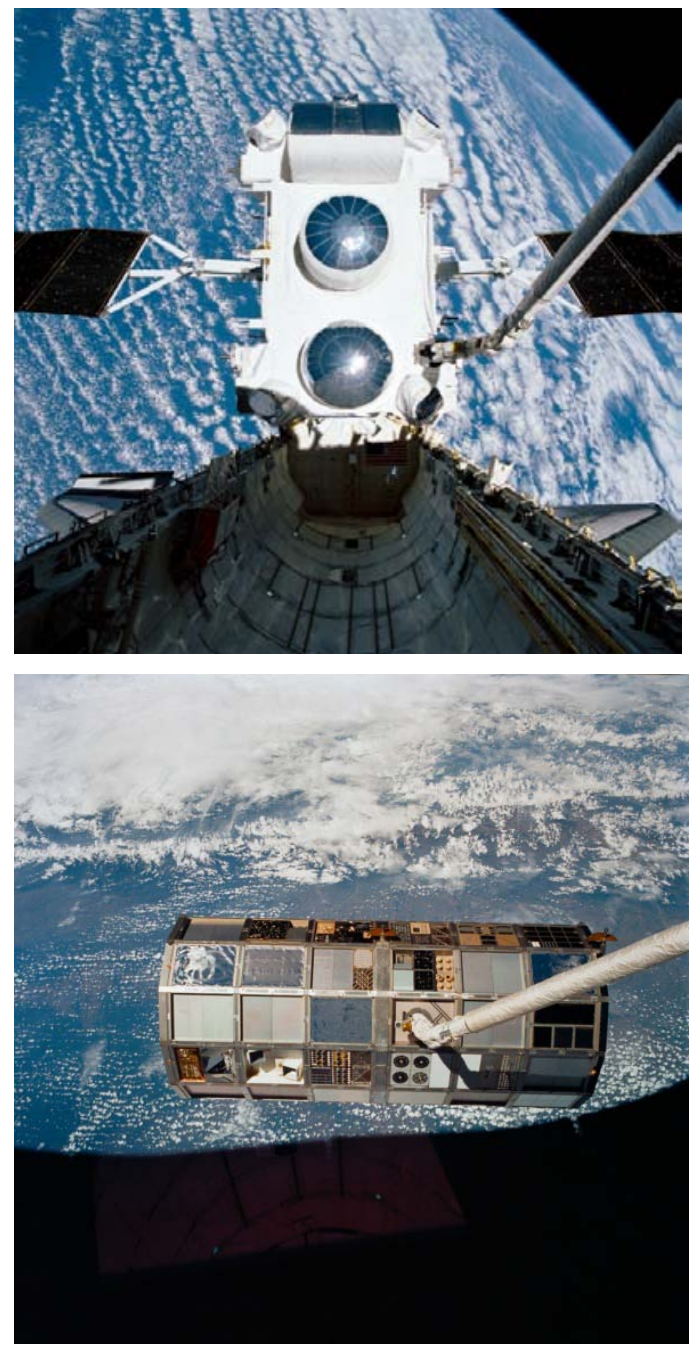


\section{SRMS History}

\section{EVA Platform}

- Developed over life of the program as an important use of SRMS for both pre-planned and contingency operations

- Crewman mounted on foot restraint at End Effector - SRMS provided a platform to react forces

- Communication from crewman to SRMS operator allowed quick and easy positioning

- Tool stanchions and holders developed to aid in repairs

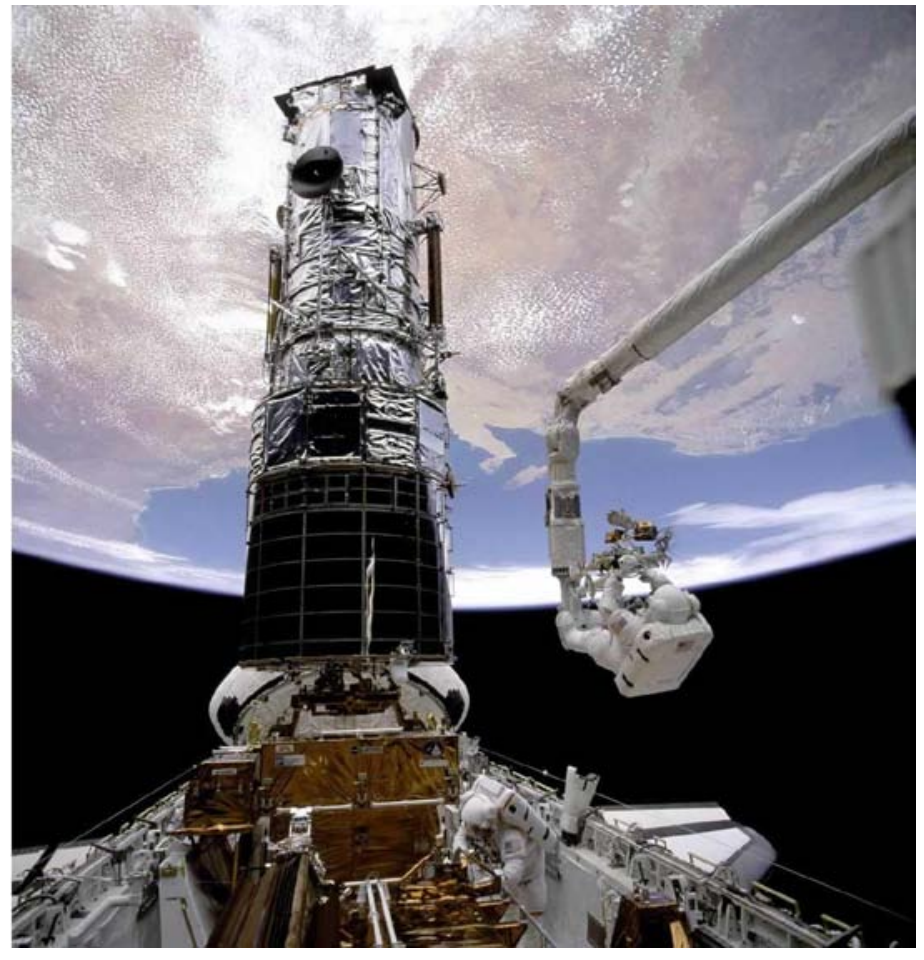

STS-61 EVA Hubble Repair 


\section{SRMS History}

\section{Space Station Construction}

- Primary mechanism used for early ISS construction

- Nodes

- US Lab

- Truss segments

- Russian Zarya Module

- Later stages - used with the SSRMS to complete construction

- Servo Electronics upgraded to improve controllability of heavier payloads

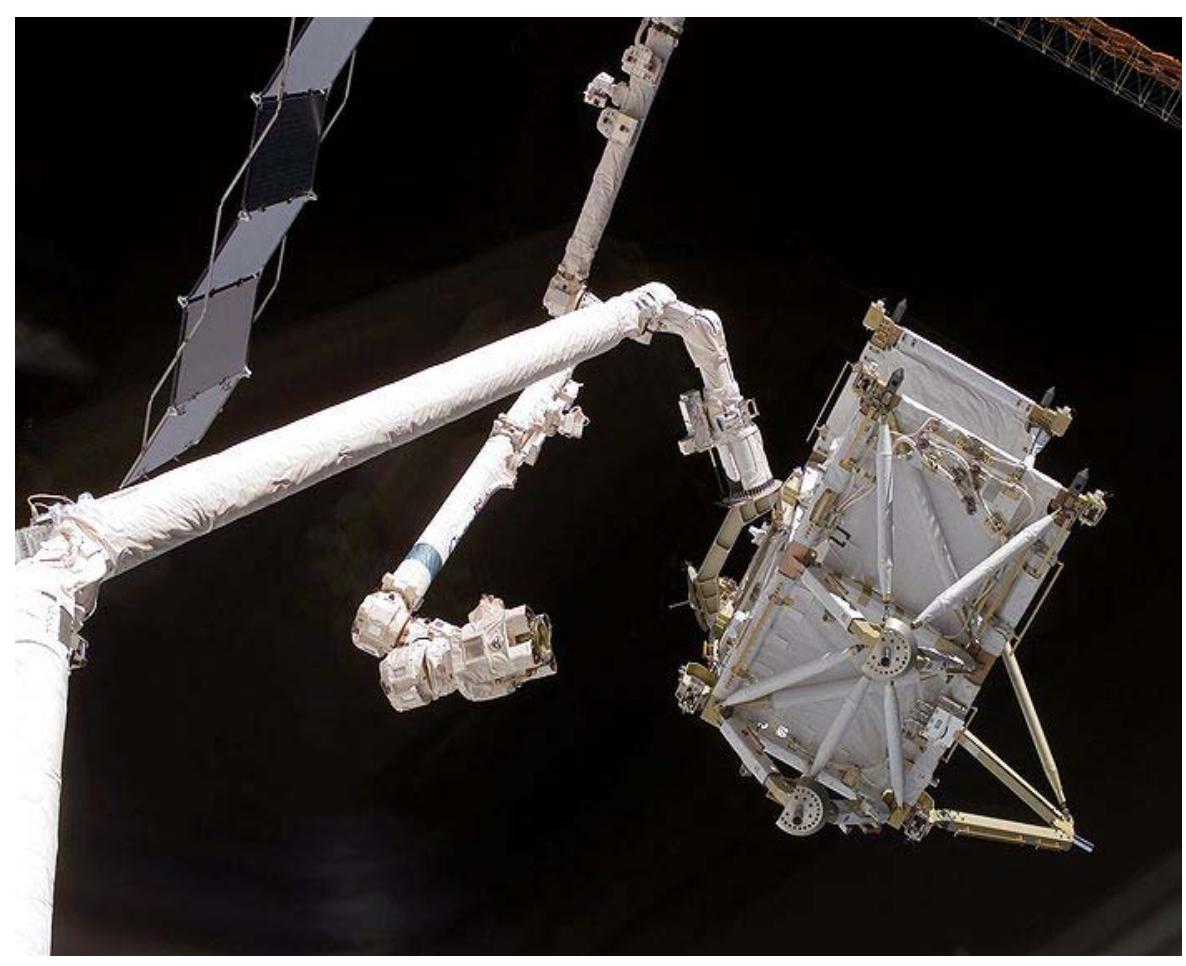

P5 Handoff from SRMS to SSRMS 


\section{SRMS History \\ Post Columbia - The Orbiter Boom Sensor System}

- OBSS developed to scan the Shuttle Thermal Protection System

- 50 foot boom with Cameras and Laser Sensors

- Robotically compatible with the SRMS - Electrical connector on End Effector provided path for power and data to Sensors

- EVA repair of TPS - 2 Scenarios

- Orbiter Repair Maneuver - SRMS grapples ISS and moves Orbiter to position where EVA on SSRMS can repair

- Crewman on the OBSS - DTO on STS-121 showed feasibility of repair with crewman on OBSS

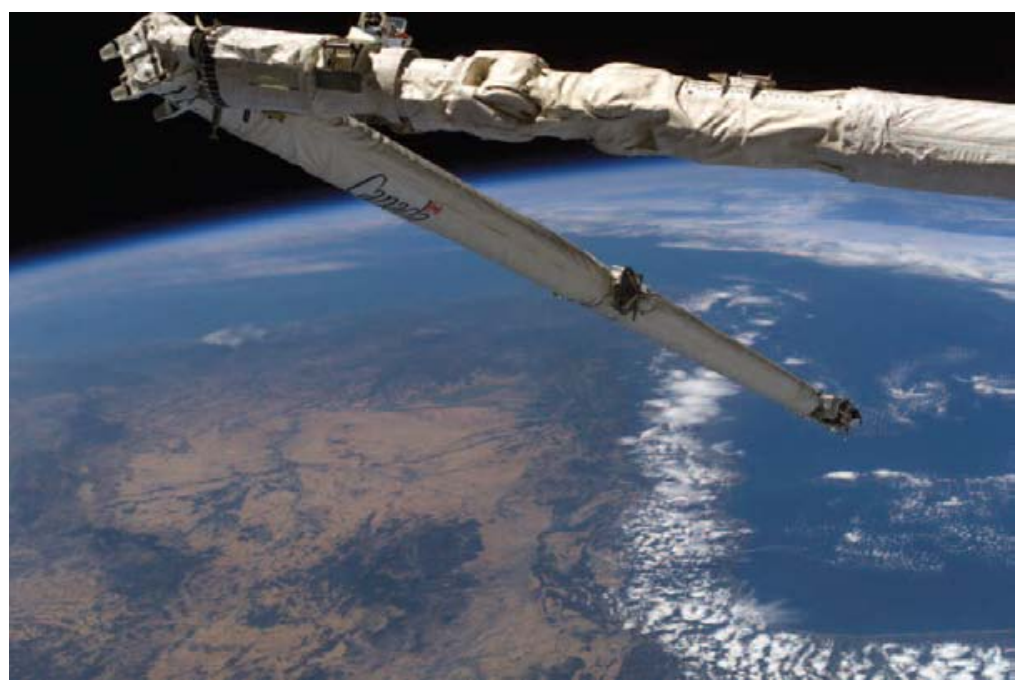

OBSS Grappled by SRMS 


\section{Unique Challenges}

Test and Verification - Air Bearing Test Rig

- 1-G environment required unique test method to verify multi-joint point of resolution control

- Air Bearing test rig developed to allow motion in pitch plane or yaw plane

- Parameters Verified:

- Straight path Arm Control

- Tip accuracy

- Tip force

- Response to misalignments

- Current Limit testing

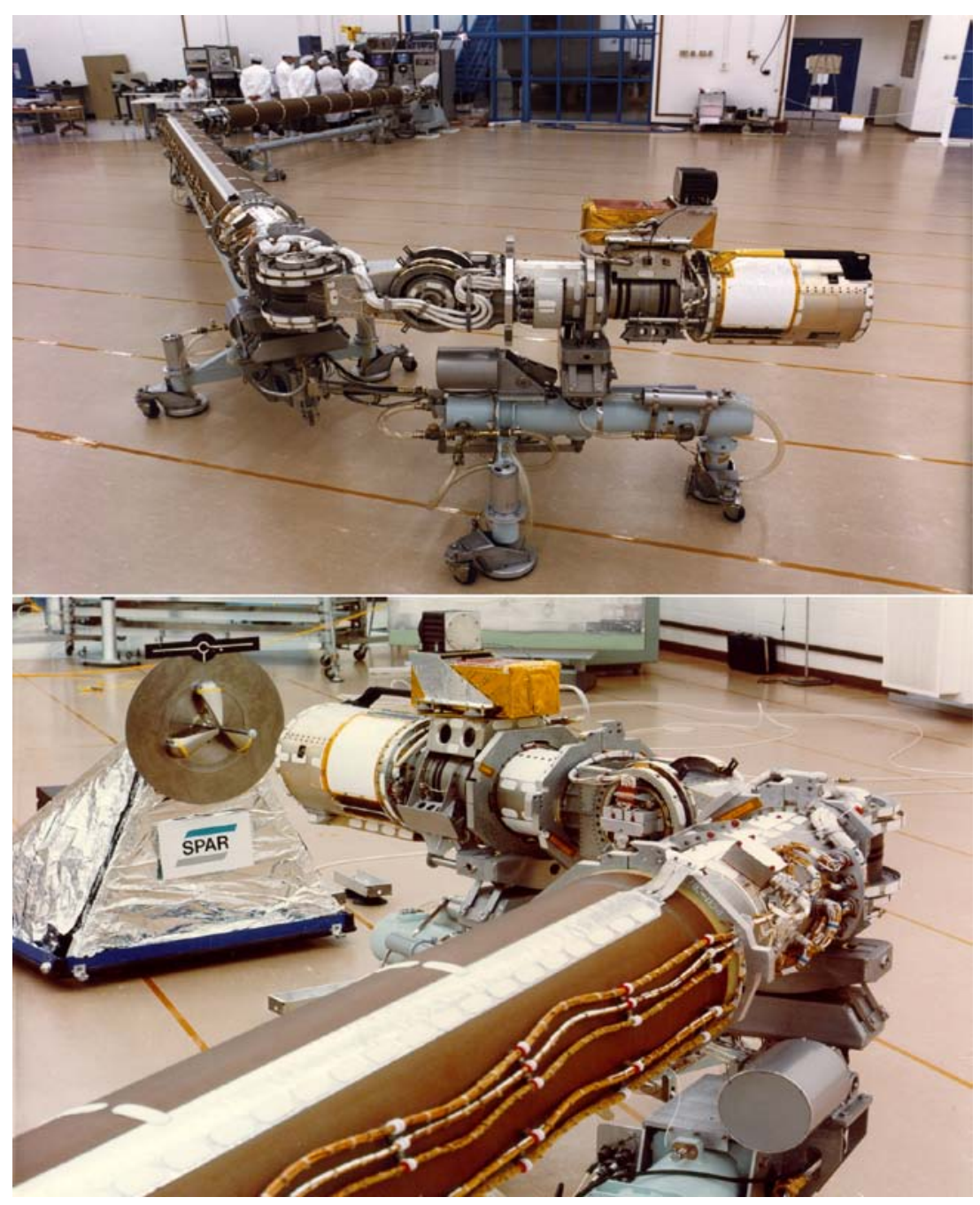




\section{Lessons Learned Enhanced Instrumentation}

\section{History}

- SRMS electronics provided motor rate and joint position data to operator and down listed to ground

- Upgrade electronics added motor current data (down listed at 1 Hertz only)

- STS-2,3 ,4, 7 and 8 had a set of strain gauges that collected loads data (Development Flight Instrumentation) - this was of limited usefulness due to the state of the technology

- Low resolution

- Poor calibration

- Difficult to retrieve data

- Limited number of payloads - no heavy payloads

- No constrained motion data

- Wireless Strain Gauge Instrumentation System installed on STS121 - DTO 849 tested SRMS/OBSS response to crewman applying loads to simulate a repair - limited data 


\section{Lessons Learned Enhanced Instrumentation}

Consequences of limited loads data

- difficulty in validating simulation models

- Tremendous effort spent in developing models and running simulations over the life of the program

- Several models developed by different organizations that did not match - truth model was difficult to find

- Life assessment was difficult

- Due to uncertainty of models - conservative assumptions had to be made 


\section{Lessons Learned Enhanced Instrumentation}

- Lesson: Implement force and moment sensors in future space robotic systems

- Benefits:

- Accurate model development

- Less analysis required - more confidence in simulations leads to less conservatism

- Better assessment of Arm capability - payload handling, constrained motion operations, life

- Enables Force Moment Accommodation to be implemented 


\section{Lessons Learned Force Moment Accommodation}

- SRMS designed for coordinated joint rate control only

- Rate control not possible during constrained motion operations - results in un-commanded motion of arm tip

- Lack of FMA noticeable during ISS construction

- Alignment difficulties during mating of modules sophisticated visual cues required

- Pre-flight analysis of load conditions during mating was required to ensure structural loads were not exceeded 


\section{Lessons Learned Force Moment Accommodation}

- Lesson: Implement FMA in future robotic systems

- Benefits:

- Better positioning accuracy during mating operations - less reliance on visual cues

- Adaption to external contact forces - lower loading conditions and reduces need for pre-mission analysis

- Ability to activate berthing and latching devices by applying pre-determined forces in commanded direction

- Complex satellite servicing tasks can be performed; refueling, module change outs, connector mate/de-mates 


\section{Unique Challenges: Software Singularity Management}

- There are 3 SRMS control singularities due to zeroes in the joint rate to tip rate Jacobian

- Shoulder singularity: wrist yaw joint directly over shoulder joint no $+/-y$ translation control

- Elbow singularity: all pitch joints are colinear: no +x control

- Wrist singuarity: wrist yaw joint at $+/$ 90': limited roll control

- Software control may cause unexpected motion

- Shoulder singularity: 3 feet from Wrist yaw directly over shoulder yaw, wrist yaw is reconfigured to opposite side of singularity zone

- Elbow singularity: No change in motion

- Yaw singularity: roll motion continues at a reduced rate
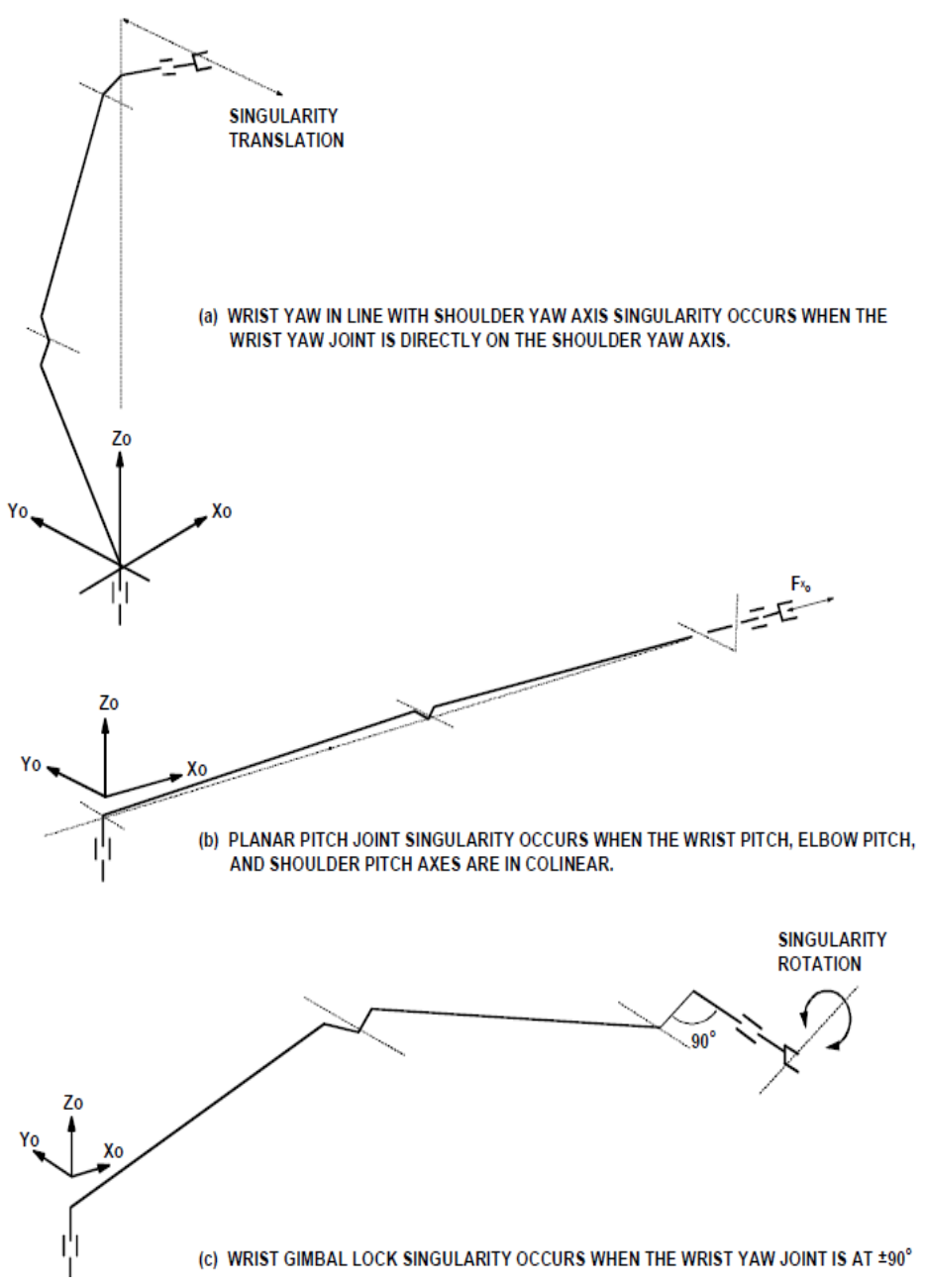


\section{Unique Challenges: Software Consistency Check}

- Runaway of an SRMS joint due to a fault that applies unlimited current to the motor would potentially drive a payload through orbiter structure and required special protection

- No runaway ever occurred during the 30 year Shuttle program

- The software consistency check monitored actual motor rate for each joint against an envelope around the motor rate command

- If the actual motor rate for any joint was outside its envelope for more than 4 consecutive GPC cycles (to allow for motor dynamics), the SRMS brakes are applied

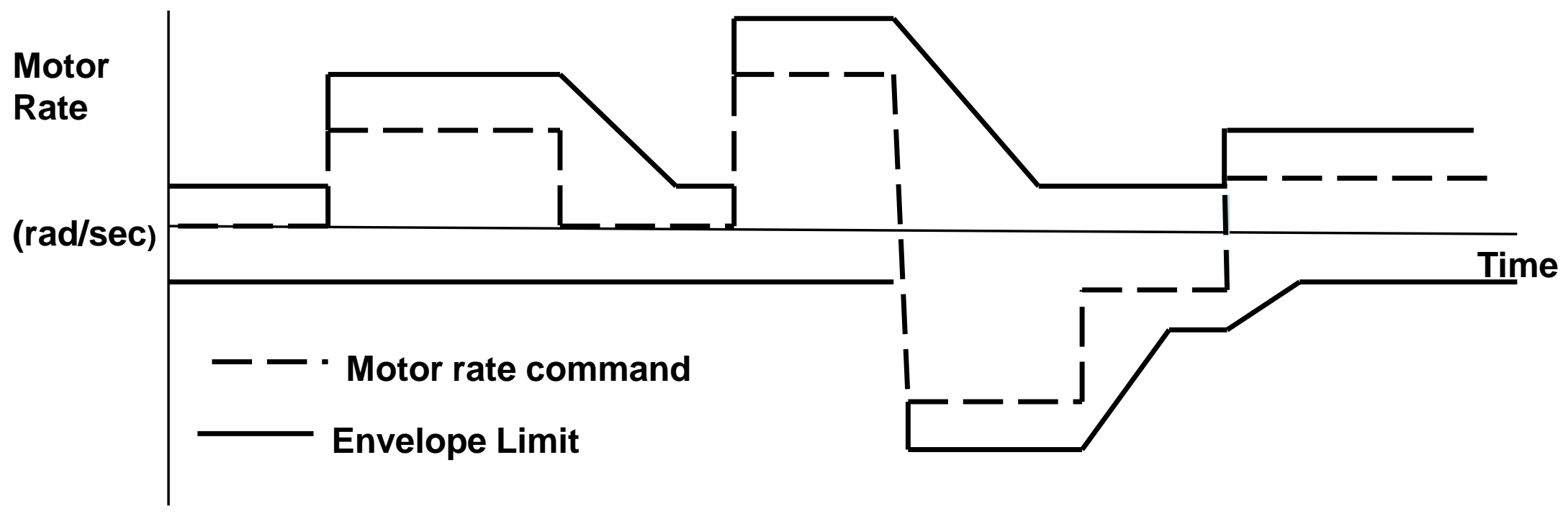




\section{Unique Challenges: Simulation}

- Simulation provided solution to inability to fully test SRMS in 1-g but also provided challenges with computers of '70's and 80's

- Real-time simulation computers did not have both speed and high precision required by SRMS model; approximations eliminated in 2004

- Testing flight software running in Orbiter computers required special purpose hardware boxes to connect Orbiter computer with simulated SRMS

- JSC's Systems Engineering Simulator (SES) combined SRMS simulation with 6 DOF dynamic motion of two vehicles

- Vehicles subjected to orbital mechanics, aerodynamics, orbiter jet thruster firings, payload control system, RCS plume impingement, berthing contact forces

- $\quad$ Functionally equivalent orbiter aft cockpit mockup provides displays and controls for SRMS and for orbiter Digital Autopilot (DAP)

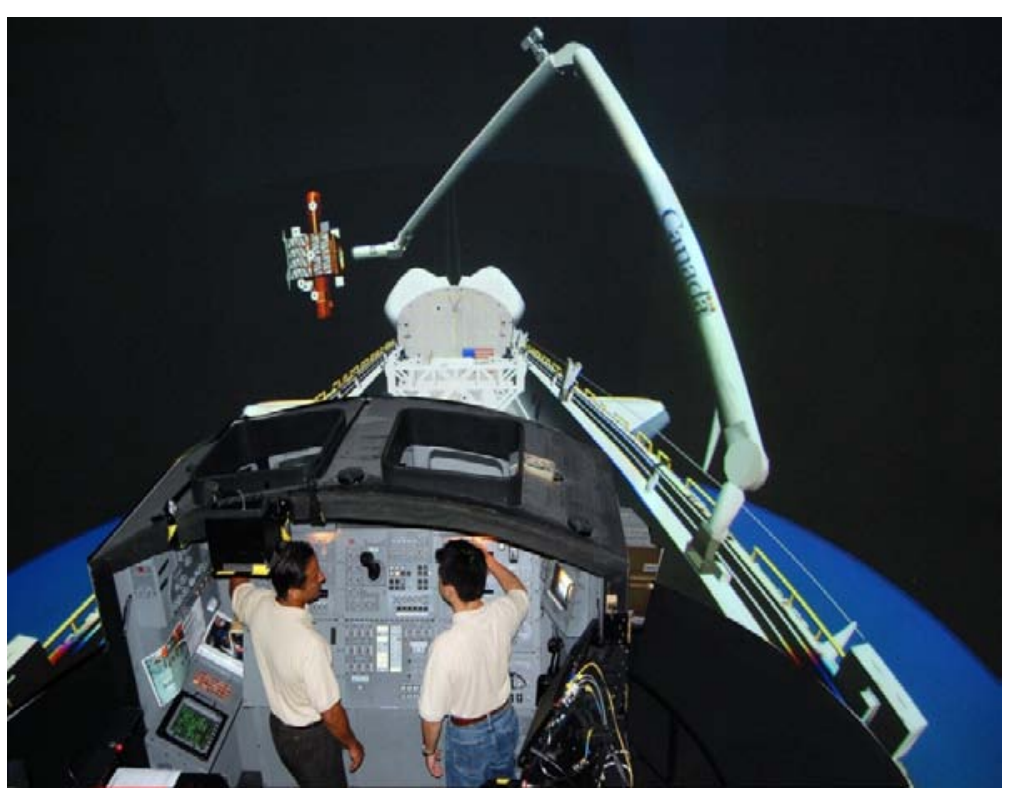

- Scene generators provide out the window and CCTV views 


\section{Unique Challenges: Simulation}

\section{Uses of SES}

- SES provided capability to test SRMS operations that require operator in the loop and also integrated SRMS and Orbiter DAP operations (such as approach to free flying payload and capture with SRMS)

- SES was used throughout the Shuttle program for engineering evaluation and also used by Mission Operations personnel for procedures development and crew training

- Uses of SES included assessment of:

- Procedures for operations on early flights that tested SRMS

- Track and capture of first payload released and recaptured by SRMS and of rotating payloads

- Uncommanded motion and software used to control it

- Berthing payloads to various latching mechanisms on ISS and shuttle

- Proposed Orbiter repair techniques

- Interaction of DAP firings in different modes and SRMS holding various payloads 


\section{Lessons Learned: Position Orientation Hold Submode}

\section{The Problem}

- The SRMS was initially purely a rate command system

- Early operators noted uncommanded motion that required significant correction commands

- During LDEF unberthing on STS-41C, crew did not correct uncommanded motion during 11 inches of unberthing $(\mathrm{z})$ motion

- Most significant uncommanded motion was 2 inches $x$ and 1 deg roll

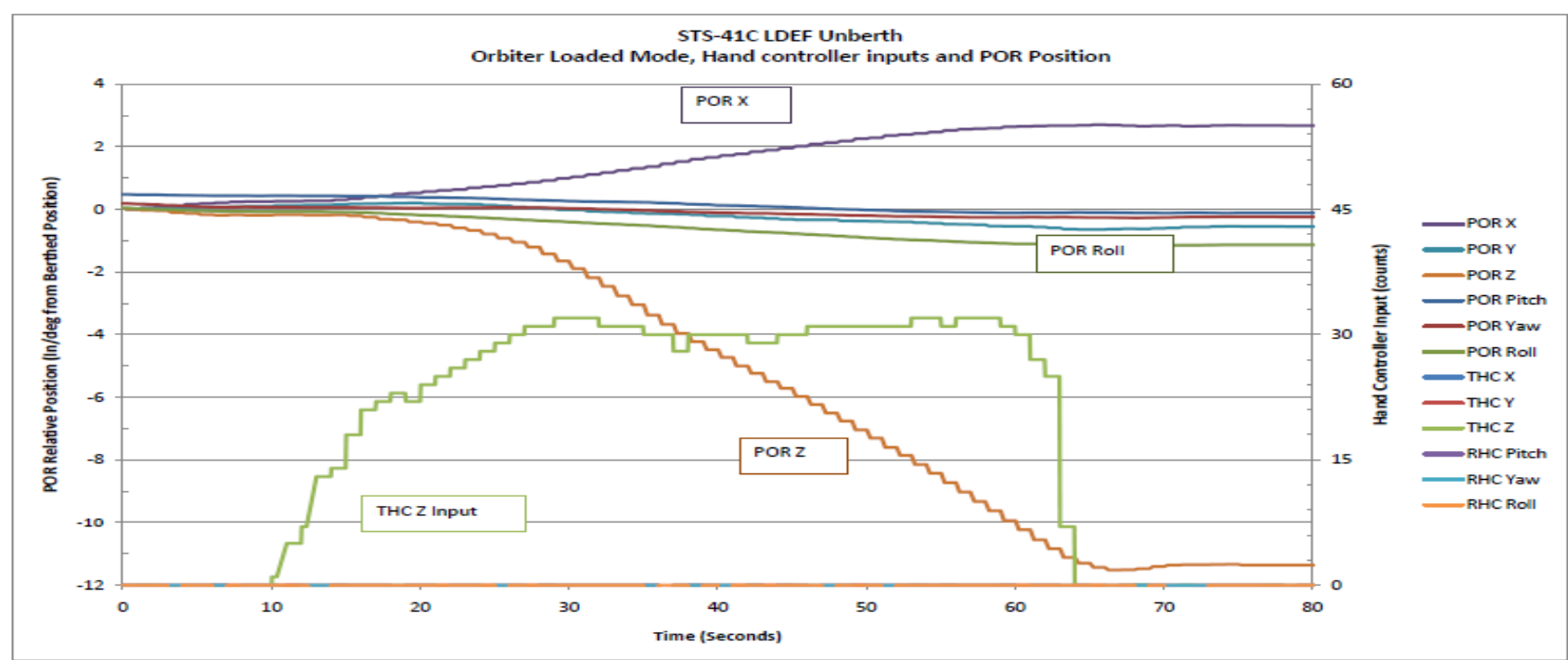

- In the early 90's, when large telescopes were unberthed the uncommanded motion became very objectionable 


\section{Lessons Learned: Position Orientation Hold Submode}

The Solution

- POHS software was developed that added position correction commands to the rate commands, based on the actual motion of the commanded point

- Initial position and command used to generate projected trajectory

- At each computer cycle, actual position compared to projected position and error perpendicular to reference trajectory used to generate POHS commands to bring actual position back to projected position

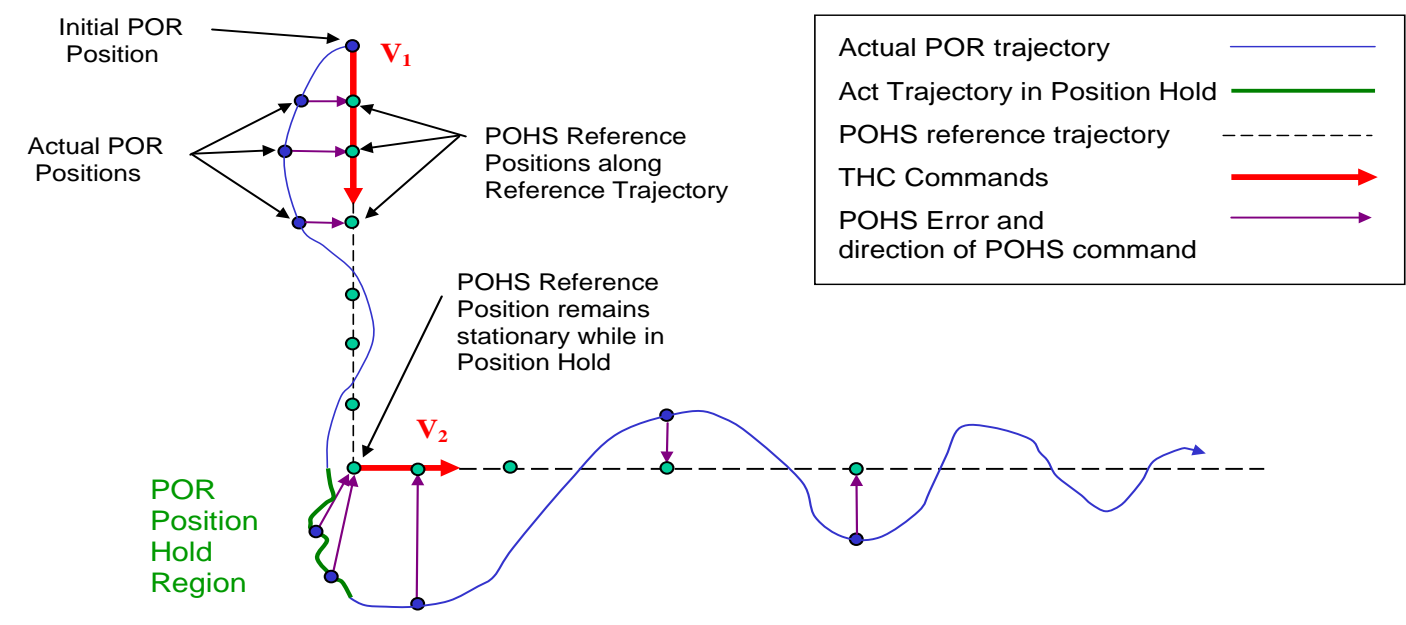

- The following charts show the difference between the translation/rotation commands and motion for Hubble Space Telescope unberthings on STS31 (no POHS) and STS-103 (POHS active) 


\section{Lessons Learned: \\ Position Orientation Hold Submode}
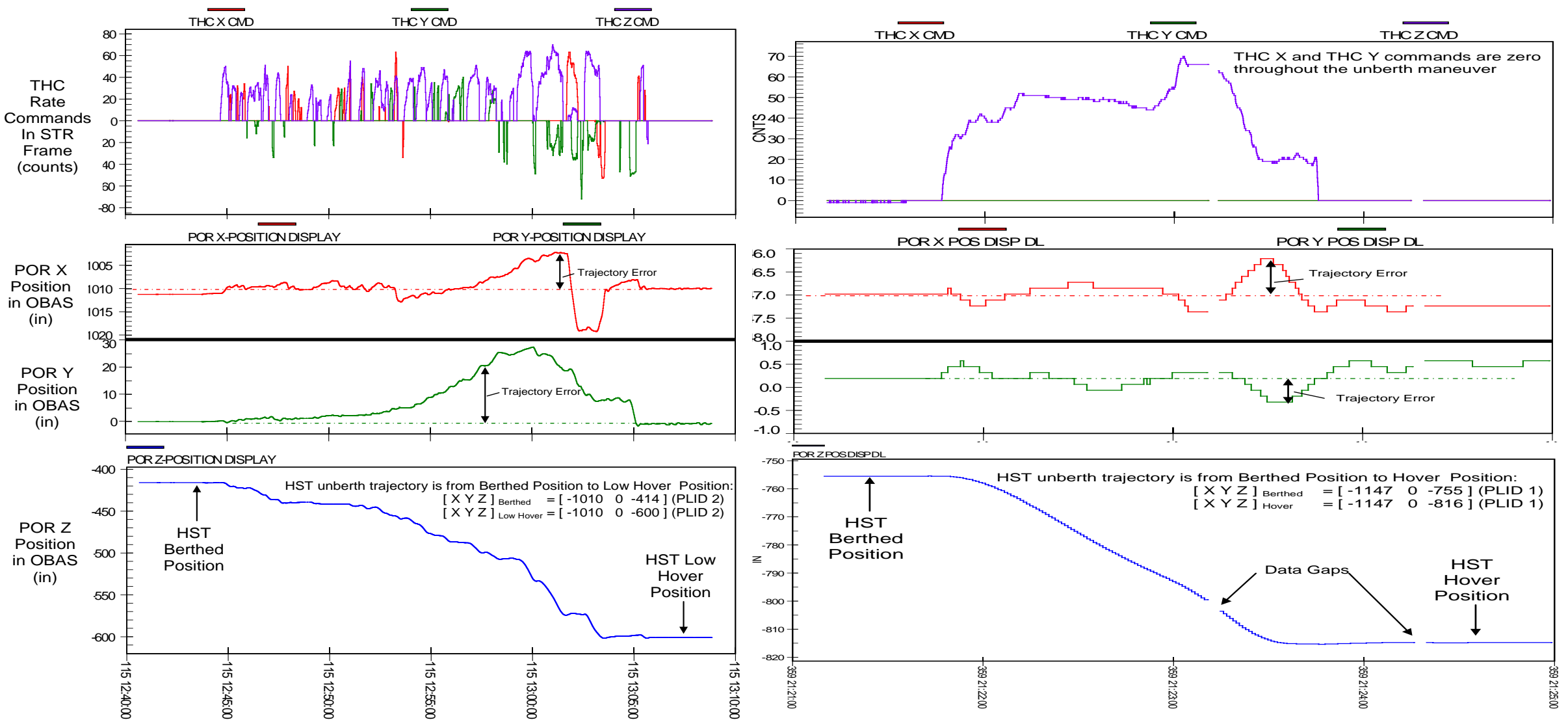

SRMS translation commands and command point motion for STS-31 (left) and STS-103 (right) 


\section{Lessons Learned: Position Orientation Hold Submode}

\section{Uses of POHS}

- POHS was initially developed for manual modes to alleviate operator workload controlling uncommanded motion, as shown in STS-31 and STS-103 unberthings

- STS-31 had multiple rotation and translation commands during unberthing

- STS-103 had one roll command before the unberthing command; no commands in other axes during unberthing

- Uncommanded motion on STS-31 (no POHS) was over 25 inches and almost 10 deg; for STS-103 (with POHS) it was less than 1 inch and about $1 / 2$ deg.

- After Columbia accident when OBSS was used in auto mode to inspect Orbiter tile and crew had limited visibility of OBSS under orbiter, POHS was added to auto modes

- $\quad$ POHS was very valuable during unberthing of large ISS modules and truss segments for ISS assembly

- Many cases of close clearance with OBSS or other elements in the Orbiter Payload Bay during unberthing 


\section{Lessons Learned: Command Wash-in in Auto Mode}

- SRMS Auto mode commands a constant rate from start point to near end point

- Command washes out to prevent overshoot of end point and rate oscillations

- After Columbia accident wash-in was also proposed for start

- Rate oscillations at start forced reduction in commanded rate so oscillations did not exceed sensor limits for damage detection

- NASA elected to reduce commanded rate instead

- Bottom figure compares rate oscillations with and without wash-in for 2.4 and 0.6 meters per minute
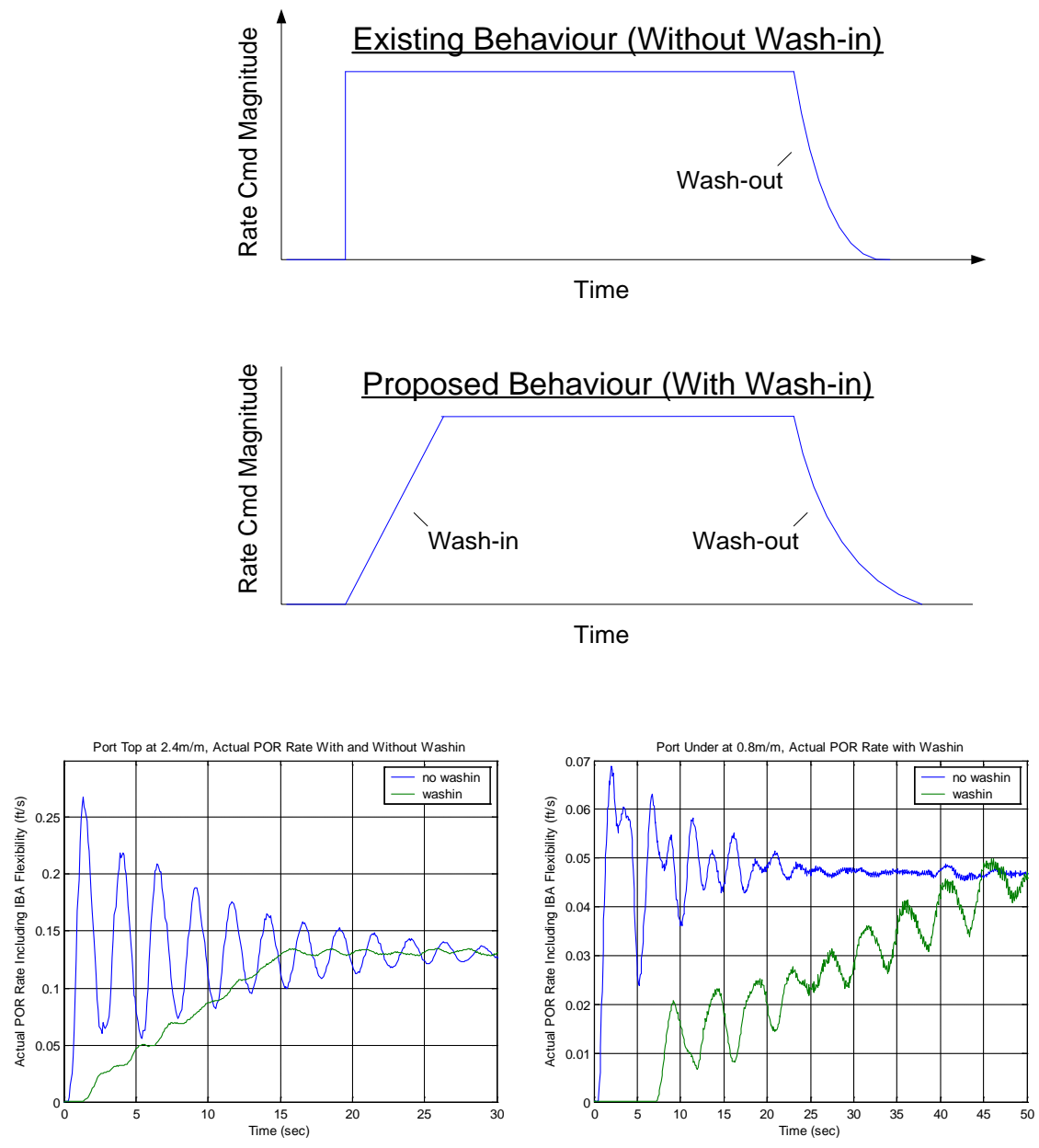


\section{Lessons Learned: Value of Open Review of Simulation Models}

\section{History}

- When the SRMS program started, modeling a 6 DOF space based robotic system was a technical challenge for all organizations

- Multiple organizations developed models independently

- Model developed by hardware provider defined as "truth" model but considered proprietary so model and implementation not disclosed

- Responses of models had differences

- With each other

- With truth sim

- All sims, including truth sim, had about equal errors with:

- Ground test data

- Flight data

- Accepted model only defined after SRMS Program Office required development of "common" model and chartered MMWG to facilitate open discussion of model and implementation details (completed 2004) 


\section{Lessons Learned: Value of Open Review of Simulation Models}

Consequences of limited insight into proprietary model

- 20 years of discussion of model/response differences at different forums without resolution

- Operations designed conservatively to cover model uncertainties

\section{Lesson Learned}

- Models used by a program should be open for review and well grounded with ground test data and flight data

\section{Benefits}

- Avoids duplication except when necessary (necessary if modeling a system at the limit of standard practice)

- Less time trying to understand differences

- Accurate model developed early in program 


\section{Lessons Learned: Load Relief in Space Based Robotics \\ History}

- SRMS has scaled brakes: weaker at wrist, stronger at elbow, strongest at shoulder

- Wrist brakes slip under high load and orbiter does not see high loads at longeron

- SSRMS design choice was to use equal strength brakes at all joints

- Provides inchworm capability

- Requires operational workaround to avoid potential high loads if brakes come on while berthing mechanism is pulling element held by SSRMS

- Proposed design to allow both inchworm capability and load relief would use two brakes at end joint and reduced strength brakes at elbow

- First brake scaled to provide load relief when that end is used at tip

- Second brake adds torque to make total torque adequate for shoulder

- Requires positive lockout of second brake at tip joints

- Dual brakes add mechanical complexity and weight

- Need to weigh against gain in load relief, if important 